\title{
Studies on the Baylis-Hillman reaction of pyrazolecarbaldehydes under the influence of DABCO: positional effect on the reactivity of the formyl group
}

\author{
Somnath Nag, Virender Singh, and Sanjay Batra* \\ Medicinal and Process Chemistry Division, Central Drug Research Institute, PO Box 173, \\ Lucknow 226001, India \\ E-mail: batra_san@yahoo.co.uk
}

\begin{abstract}
Results of the study to assess the difference in reactivity of the formyl group present at various positions in substituted pyrazolecarbaldehydes for the Baylis-Hillman reaction under the influence of DABCO are described.
\end{abstract}

Keywords: Baylis-Hillman reaction, DABCO, pyrazolecarbaldehydes, formyl group reactivity

\section{Introduction}

The Baylis-Hillman reaction has been instrumental in providing ready access to several useful intermediates, which lead to generation of novel structural motifs. ${ }^{1}$ The varying conditions ${ }^{2}$ and development of novel catalysts ${ }^{3}$ for this particular reaction have not only provided new alternatives to synthetic chemists but has also led to considerable progress in understanding of the mechanism of this reaction. ${ }^{4}$ Despite this enormous development, not much attention has been directed towards understanding the difference in reactivity of the formyl group present at various positions within a system. In case of substituted benzaldehydes it is well known that the substitution on the benzene ring has direct implications on the reactivity of the formyl group. The nitro- or trifluormethyl-substituted benzaldehydes are some of the fastest reacting substrates due to their electron-withdrawing character while the methoxy- or dimethoxybenzaldehyde's sluggish nature for the Baylis-Hillman reaction is attributed to their electron-donating nature. However, in case of heterocyclic systems such effects are not pronounced and therefore not much has been described pertaining to the difference in reactivity of the formyl group present at different positions within a heterocyclic system for this reaction. We were the first to examine the difference in reactivity of the formyl group for the Baylis-Hillman reaction in various substituted isoxazolecarbaldehydes. We have observed that the formyl group present at 3 or 5position in isoxazole ring undergo a fast Baylis-Hillman reaction as compared to the formyl 
group present at the 4-position (Fig. 1). ${ }^{5}$

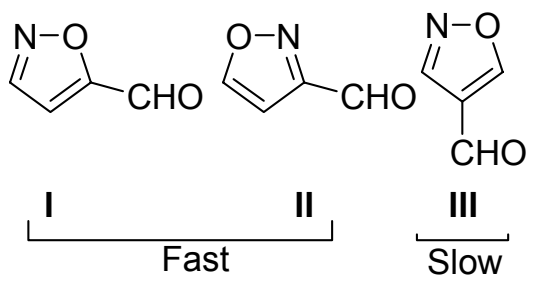

Figure 1. 5-(I) and 3-isoxazolecarbaldehyde (II) are fast reacting substrate for Baylis-Hillman reaction while 4-isoxazolecarbaldehyde (III) is a slow reacting one under similar conditions.

The better reactivity of 3- and 5-isoxazolecarbaldehydes was attributed to a hypothetical assumption that the lone pair of electrons in the heteroatoms present in proximity to the carbon atom bearing the formyl group helps in eliminating the tertiary base from the intermediate in the final step of the reaction. However, possibility of other electronic or steric factors or the effect of the basicity of the heteroatom present in the proximity of the carbon bearing formyl group is not ruled out. We have also reported that the presence of a electron withdrawing group such as methoxy carbonyl on the carbon adjacent to the one bearing the formyl group further increases its reactivity for this particular reaction. ${ }^{6}$ Recently, Rao et al. ${ }^{7}$ reported that substituted 2-chloro3-pyridinecarbaldehydes are also fast reacting substrate for Baylis-Hillman reaction. Although they did not draw any comparison of their substrate with 3-pyridinecarbaldehyde, it can be extended from the literature that perhaps the formyl group in 2-chloro-3-pyridinecarbaldehyde have better reactivity than the 3-pyridinecarbaldehyde. It is quite likely that the presence of a halide on the carbon adjacent to the one bearing the formyl group may have some effect on its reactivity for the Baylis-Hillman reaction. These observations provoked the need to explore the reactivity of the formyl group towards this reaction in other heterocyclic systems too. In our studies aimed at this objective we have carried out the Baylis-Hillman reaction of differently substituted pyrazolecarbaldehydes. This has led to interesting results which prompt us to report the details of our study in this paper.

The choice of studying the pyrazolecarbaldehyde was influenced by the fact that it is one of the closest heterocyclic systems to isoxazolecarbaldehydes and desired substrates can be easily generated. Moreover, Baylis-Hillman derivatives of different pyrazolecarbaldehydes shall lead to generation of several building blocks which can be synthetically diversified to obtain the chemical libraries of pyrazoles. Several pyrazole derivatives are of high pharmaceutical interest and are ascribed with wide range of bioactivities. ${ }^{8}$ These include antirheumatic, topoisomerse inhibitors, antiinflammatory, anticoagulant, cannabinoid receptor ligands, antimicrobials and estrogen receptor antagonists. 


\section{Results and Discussion}

The initial objective to generate different pyrazolecarbaldehydes was achieved either by known procedures or via modifying the literature methodologies. The scheme 1 illustrates the synthesis of 1,5-disubstituted-1 $\mathrm{H}$-pyrazole-3-carbaldehyde and 2,5-disubstituted-2H-pyrazole-3carbaldehyde. ${ }^{9}$ The treatment of acetophenones 1a-c with diethyl oxalate in the presence of $\mathrm{NaH}$ furnished the diketoesters 2a-c. Reaction of 2a-c with phenylhydrazine in the presence of catalytic amount of TFA yielded a mixture of pyrazole esters 3a-c and 4a-c. Separation of these regioisomers at this stage was not convenient. Therefore the mixture containing both regioisomers was treated with $\mathrm{LiAlH}_{4}$ in dry diethylether to afford the product as mixture of 5a-c and 6a-c. Due to significant difference in the polarity of these alcohols they were easily separated via column chromatography over silica gel. Compounds 5a-c were obtained as the major isomer while 6a-c were separated as the minor product. Subsequently these alcohols were subjected to oxidation in the presence of PCC. Oxidation of alcohols 5a-c yielded 7a-c in 55$57 \%$ yield while oxidation of $\mathbf{6 a - c}$ afforded $\mathbf{8 a - c}$ in $75-80 \%$ yield.

In the next step the major products 7a-c were subjected to Baylis-Hillman reaction with several alkenes in the presence of DABCO under neat condition. Initially the reactions were carried out in the presence of 0.5 equiv. of $\mathrm{DABCO}$ and 1.2 equiv. of activated alkene (scheme 2). The results of the Baylis-Hillman reaction of 7a-c with different alkenes are summarized in Table 1. It was observed that when acrylonitrile, methyl acrylate or ethyl acrylate were employed as alkene, the starting aldehydes were completely consumed however in the case of tert-butyl acrylate or cyclohexen-1-one, some amount of starting substrate left unreacted even after 3 days.

It was interesting to note that amongst different aldehydes used for the reaction the one bearing unsubstituted phenyl group was sluggish towards the reaction as compared to the ones bearing substituted phenyl groups. It was observed during these reactions that the acrylonitrile reacts more efficiently as compared to other alkenes. Since it is known that rate of the BaylisHillman reaction is increased under the influence of sonication, ${ }^{10}$ in a model study the reaction of compound $\mathbf{7 b}$ was performed with acrylonitrile and methyl acrylate under sonication. Interestingly the reaction with acrylonitrile was complete in $3.5 \mathrm{~h}$ instead of $7 \mathrm{~h}$ while the reaction with methyl acrylate was accomplished in $8 \mathrm{~h}$ instead of $10 \mathrm{~h}$. These results inferred that the sonication does have positive effect on the rate of the Baylis-Hillman reaction in these substrates.

In the next stage, compounds 8a-c belonging to other regioisomer were subjected to the Baylis-Hillman reaction with several alkenes, the results of which are summarized in Table 2 (scheme 3). Interestingly unlike 7a-c, these substrates undergo faster Baylis-Hillman reaction though the behavior of different alkenes remains the same. Herein too the starting substrate failed to react completely with tert-butyl acrylate, methyl vinyl ketone or cyclohexen-1-one. Although methyl vinyl ketone is highly activated alkene, the incomplete reaction may be speculated due to Michael type dimerization of this reagent under the influence of DABCO. ${ }^{11}$ Within this prototype, different aldehydes had almost similar reaction rates. 


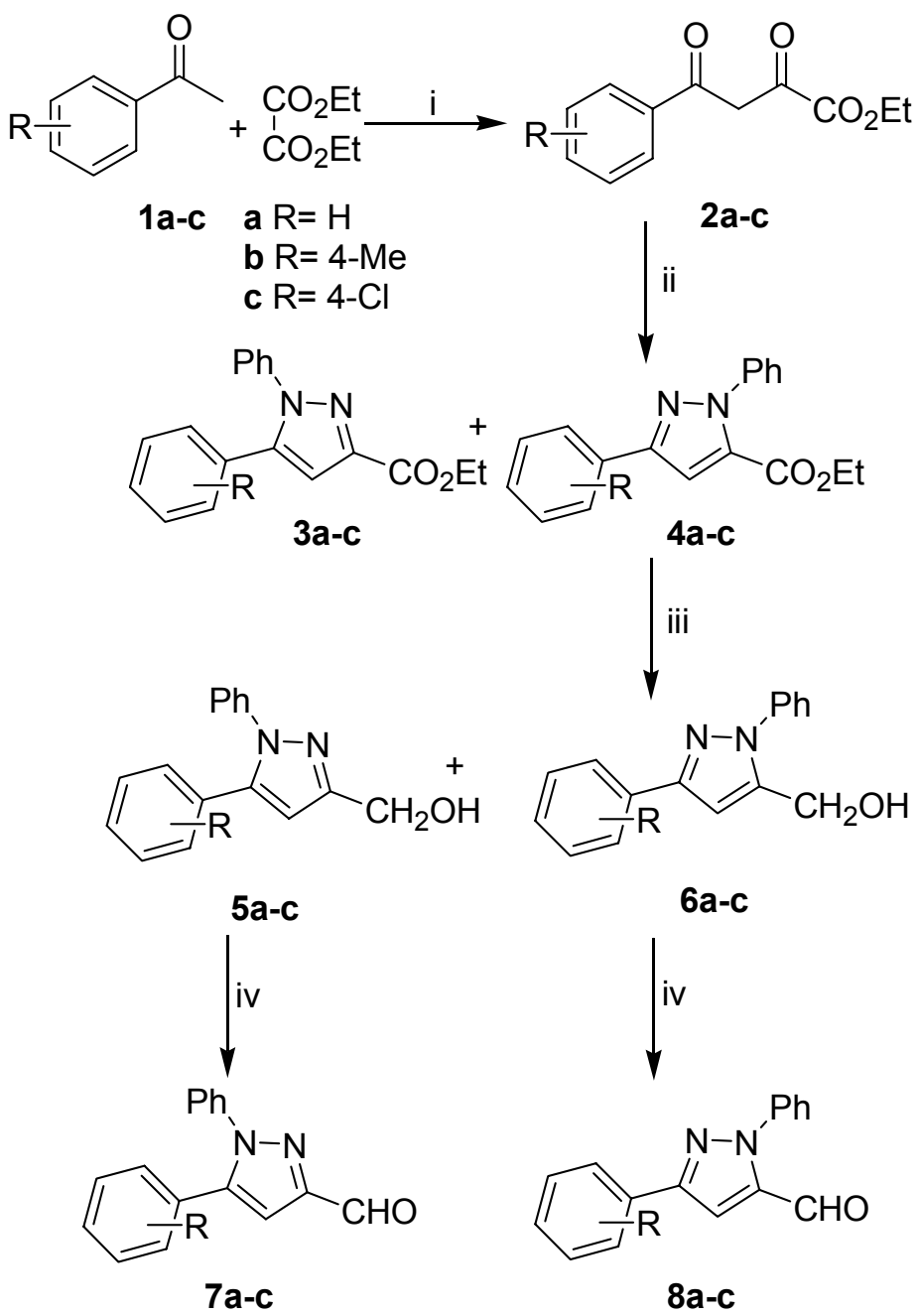

Scheme 1. Reagents and conditions: i) a) $\mathrm{NaH}$ ( $60 \%$ in oil), dry benzene, $\mathrm{rt}$ to $80{ }^{\circ} \mathrm{C}, 2 \mathrm{~h}$. b) $2 \%$ aq. $\mathrm{HCl}$; ii) $\mathrm{PhNHNH}_{2}$, TFA (catalytic), ethanol, reflux, 2h; iii) $\mathrm{LiAlH}_{4}$, dry diethylether; iv) PCC, dry $\mathrm{CH}_{2} \mathrm{Cl}_{2}$.

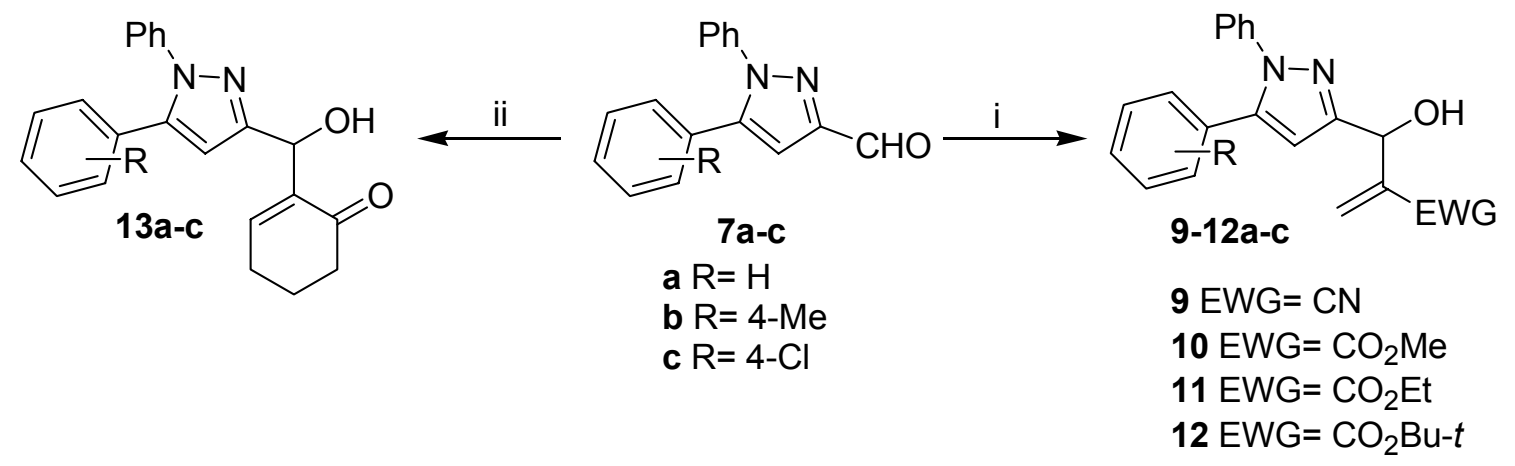

Scheme 2. Reagents and conditions: i) $\mathrm{CH}_{2}=\mathrm{CHEWG}$, DABCO, rt, for time refer to Table 1; ii) 2-cyclohexen-1-one, DABCO, rt. 
Table 1. Results of the Baylis-Hillman reaction of $7 \mathbf{a}-\mathbf{c}^{\mathrm{a}}$

\begin{tabular}{lllll}
\hline \multirow{2}{*}{$\begin{array}{l}\text { Compd. } \\
\text { No. }\end{array}$} & EWG & ${\text { Time }(\mathbf{h})^{\mathrm{b}}}$ & \multicolumn{2}{l}{ Isolated Yields (\%) } \\
\cline { 4 - 5 } & & & Product & Starting (7) \\
\hline $\mathbf{9 a}$ & $\mathrm{CN}$ & 9 & 86 & - \\
$\mathbf{9 b}$ & $\mathrm{CN}$ & 7 & 83 & - \\
$\mathbf{9 c}$ & $\mathrm{CN}$ & 6 & 88 & - \\
$\mathbf{1 0 a}$ & $\mathrm{CO}_{2} \mathrm{Me}$ & 10 & 88 & - \\
$\mathbf{1 0 b}$ & $\mathrm{CO}_{2} \mathrm{Me}$ & 8 & 79 & - \\
$\mathbf{1 0 c}$ & $\mathrm{CO}_{2} \mathrm{Me}$ & 8 & 83 & - \\
$\mathbf{1 1 a}$ & $\mathrm{CO}_{2} \mathrm{Et}$ & 22 & 81 & - \\
$\mathbf{1 1 b}$ & $\mathrm{CO}_{2} \mathrm{Et}$ & 16 & 85 & - \\
$\mathbf{1 1 c}$ & $\mathrm{CO}_{2} \mathrm{Et}$ & 16 & 84 & - \\
$\mathbf{1 2 a}$ & $\mathrm{CO}_{2} \mathrm{Bu}-t$ & $90 \mathrm{days}^{\mathrm{c}}$ & 56 & 39 \\
$\mathbf{1 2 b}$ & $\mathrm{CO}_{2} \mathrm{Bu}-t$ & 72 & 54 & 32 \\
$\mathbf{1 2 c}$ & $\mathrm{CO}_{2} \mathrm{Bu}-t$ & 72 & 58 & 36 \\
$\mathbf{1 3 a}$ & - & 80 & 61 & 14 \\
$\mathbf{1 3 b}$ & - & 72 & 71 & 10 \\
$\mathbf{1 3 c}$ & - & 72 & 67 & 11 \\
\hline
\end{tabular}

${ }^{a}$ All reactions were carried out with 0.5 eq. of $\mathrm{DABCO}$ and 1.2 eq. of activated alkene with respect to starting aldehyde under neat condition. ${ }^{\mathrm{b}}$ The time and isolated yields included in the table are mean of two set of reactions with each substrate. After the specified time no further change was observed in the reaction. ${ }^{\text {T }}$ This reaction was worked up after 90 days as no product was observed upto 7 days.

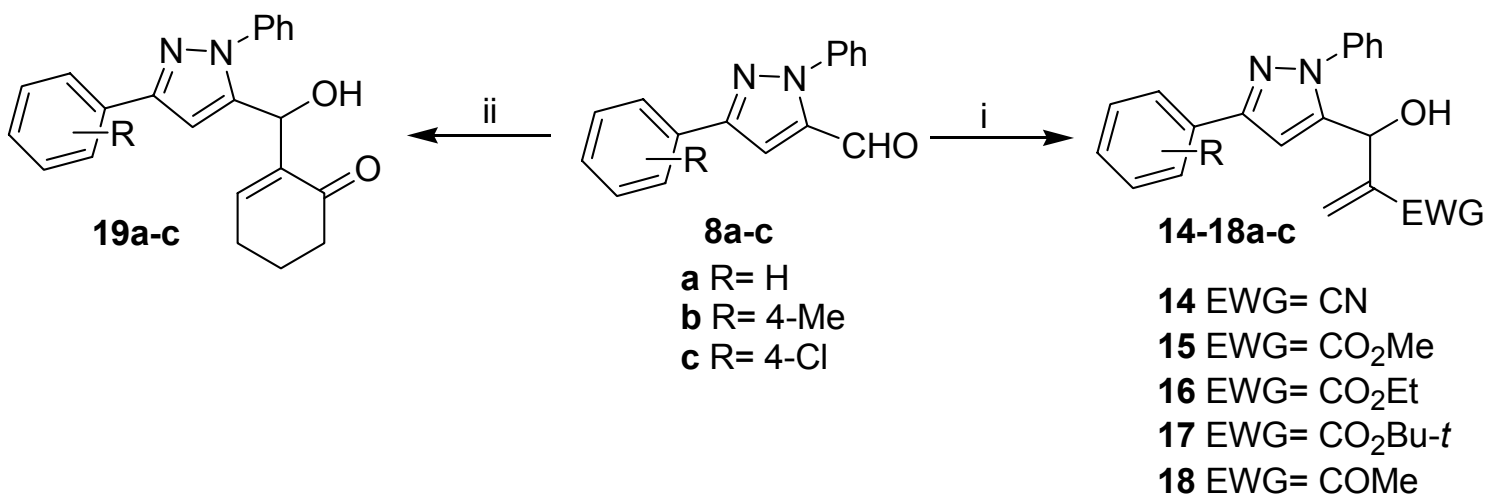

Scheme 3. Reagents and Conditions: i) $\mathrm{CH}_{2}=\mathrm{CHEWG}, \mathrm{DABCO}$, rt, for time refer to Table 2; ii) 2-cyclohexen-1-one, DABCO, rt. 
Table 2. Results of the Baylis-Hillman reaction of $\mathbf{8 a -}-\mathbf{c}^{\mathrm{a}}$

\begin{tabular}{|c|c|c|c|c|}
\hline \multirow{2}{*}{$\begin{array}{l}\text { Compd. } \\
\text { No. }\end{array}$} & \multirow[t]{2}{*}{ EWG } & \multirow[t]{2}{*}{ Time $(h)^{b}$} & \multicolumn{2}{|c|}{ Isolated Yields (\%) } \\
\hline & & & Product & Starting (8) \\
\hline $14 a$ & $\mathrm{CN}$ & 3.5 & 91 & - \\
\hline $14 b$ & $\mathrm{CN}$ & 4.0 & 83 & - \\
\hline $14 \mathrm{c}$ & $\mathrm{CN}$ & 3.5 & 78 & - \\
\hline $15 a$ & $\mathrm{CO}_{2} \mathrm{Me}$ & 5.0 & 83 & - \\
\hline 15b & $\mathrm{CO}_{2} \mathrm{Me}$ & 6.5 & 87 & - \\
\hline $15 \mathrm{c}$ & $\mathrm{CO}_{2} \mathrm{Me}$ & 5.0 & 80 & - \\
\hline 16a & $\mathrm{CO}_{2} \mathrm{Et}$ & 13 & 89 & - \\
\hline $16 b$ & $\mathrm{CO}_{2} \mathrm{Et}$ & 13 & 92 & - \\
\hline $16 \mathrm{c}$ & $\mathrm{CO}_{2} \mathrm{Et}$ & 9.5 & 84 & - \\
\hline $17 \mathbf{a}$ & $\mathrm{CO}_{2} \mathrm{Bu}-t$ & 48 & 68 & 25 \\
\hline $17 \mathrm{~b}$ & $\mathrm{CO}_{2} \mathrm{Bu}-t$ & 48 & 78 & 16 \\
\hline $17 \mathrm{c}$ & $\mathrm{CO}_{2} \mathrm{Bu}-t$ & 48 & 75 & 20 \\
\hline $18 \mathrm{a}$ & $\mathrm{COMe}$ & 6 & 56 & 32 \\
\hline $18 b$ & $\mathrm{COMe}$ & 5 & 32 & 39 \\
\hline $18 \mathrm{c}$ & $\mathrm{COMe}$ & 5 & 41 & 33 \\
\hline 19a & - & 48 & 65 & - \\
\hline $19 b$ & - & 48 & 72 & - \\
\hline $19 \mathrm{c}$ & - & 48 & 68 & - \\
\hline
\end{tabular}

${ }^{a}$ All reactions were carried out with 0.5 eq. of $\mathrm{DABCO}$ and 1.2 eq. of activated alkene with respect to starting aldehyde under neat condition. ${ }^{\mathrm{b}}$ The time and yields are mean of two set of reactions with each substrates. After the specified time no further change in the reaction was observed.

In the next part of the study it was desired to investigate the reactivity of the formyl group present at the 4-position of the pyrazole ring. On the basis of our earlier observation with different isoxazolecarbaldehydes, it was envisaged that the pyrazole-4-carbaldehyde would be a slow reacting substrate for the Baylis-Hillman reaction. Therefore, the synthesis of 4pyrazolecarbaldehydes 20a-c was accomplished via the reported strategy (scheme 4). ${ }^{12}$ Compounds 20a-c were subjected to the Baylis-Hillman reaction with different alkenes. Expectedly, the reaction failed to occur with 0.5 equiv. of DABCO and 1.2 equiv. of alkenes. Therefore, the amount of DABCO was increased to 1.0 equiv. while that of alkenes to 3.0 equiv. Results of the Baylis-Hillman reaction with 4-pyrazolecarbaldehydes are summarized in Table 3. In most of the cases the reactions take longer periods and yields of adducts were low. The unsubstituted phenyl group bearing pyrazolecarbaldehyde behaved as poor electrophile and no product could be isolated in the case of ethyl and tert-butyl acrylates. 


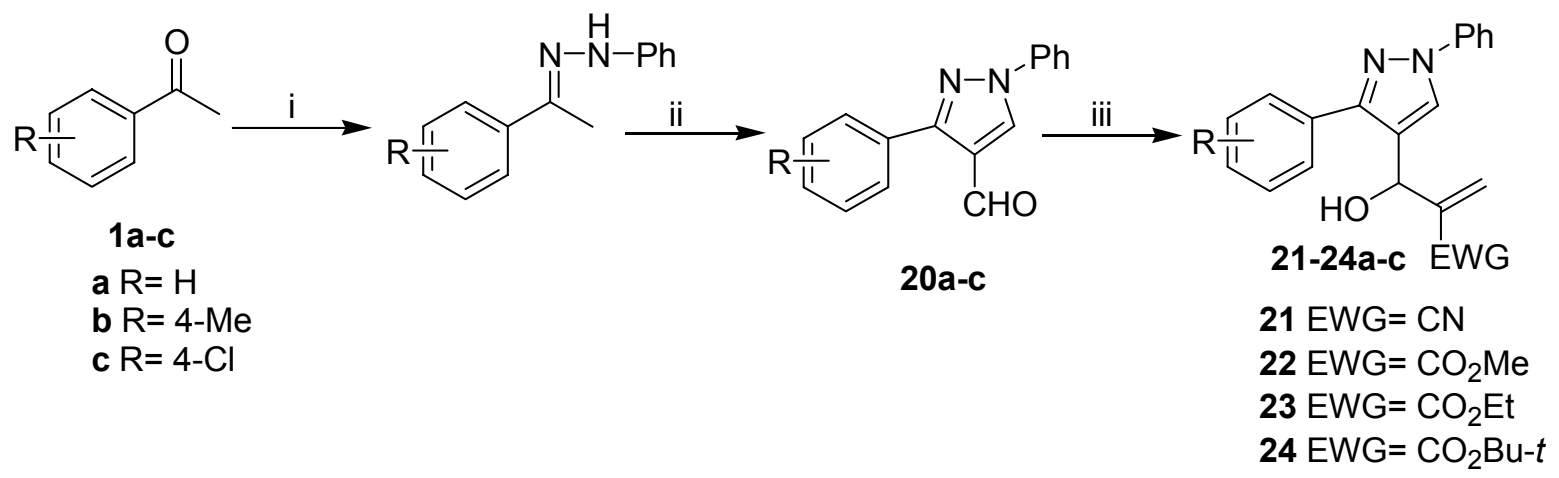

Scheme 4. Reagents and Conditions: i) $\mathrm{PhNHNH}_{2}$, pTSA (catalytic), dry toluene, reflux, 3h; ii) $\mathrm{POCl}_{3}, \mathrm{DMF}, 80^{\circ} \mathrm{C}, 2 \mathrm{~h}$; iii) $\mathrm{CH}_{2}=\mathrm{CHEWG}, \mathrm{DABCO}$, rt, for time refer to Table 3.

Table 3. Results of the Baylis-Hillman reaction of 20a-c ${ }^{\mathrm{a}}$

\begin{tabular}{lllll}
\hline $\begin{array}{llll}\text { Compd. } \\
\text { No. }\end{array}$ & EWG & Time (days) & \multicolumn{2}{l}{ Isolated Yields (\%) } \\
\cline { 3 - 5 } & & & Product & Starting (20) \\
\hline 21a & $\mathrm{CN}$ & 4 & 7 & 86 \\
21b & $\mathrm{CN}$ & 4 & 55 & 38 \\
21c & $\mathrm{CN}$ & 4 & 43 & 51 \\
22a & $\mathrm{CO}_{2} \mathrm{Me}$ & 25 & 12 & 80 \\
22b & $\mathrm{CO}_{2} \mathrm{Me}$ & 25 & 45 & 49 \\
22c & $\mathrm{CO}_{2} \mathrm{Me}$ & 25 & 16 & 80 \\
23a & $\mathrm{CO}_{2} \mathrm{Et}$ & 25 & $\mathrm{NR}$ & 90 \\
23b & $\mathrm{CO}_{2} \mathrm{Et}$ & 25 & 22 & 70 \\
23c & $\mathrm{CO}_{2} \mathrm{Et}$ & 25 & 13 & 83 \\
24a & $\mathrm{CO}_{2} \mathrm{Bu}-t$ & 25 & $\mathrm{NR}$ & 92 \\
24b & $\mathrm{CO}_{2} \mathrm{Bu}-t$ & 25 & 8 & 80 \\
24c & $\mathrm{CO}_{2} \mathrm{Bu}-t$ & 25 & $\mathrm{NR}$ & 94 \\
\hline
\end{tabular}

${ }^{\mathrm{a}}$ All reactions were carried out with 1.0 eq. of $\mathrm{DABCO}$ and 3.0 eq. of activated alkene with respect to starting aldehyde under neat condition. ${ }^{\mathrm{b}}$ The reaction was pursued for longer periods when no product formation was observed in 7 days. $\mathrm{NR}=$ no reaction

Acrylonitrile here too underwent Baylis-Hillman reaction at a better rate as compared to other alkenes. With the objective to improve the reaction rates and yields, sonication was also attempted but without any success. Additionally in a representative study the reactions of 20c with acrylonitrile were performed using methanol and DMSO as the solvent. In the presence of methanol though the reaction was clean only $18 \%$ of the product could be isolated after $96 \mathrm{~h}$ of reaction time. On the contrary the reaction in DMSO led to formation of number of spots on TLC, but a careful chromatography led to isolation of $38 \%$ of the Baylis-Hillman adduct and 
recovery of the $20 \%$ of the starting material. Further reaction of $\mathbf{2 0 b}$ with acrylonitrile in the presence of trimethylamine under aqueous methanol furnished only $15 \%$ of the corresponding adduct $21 \mathrm{~b}$ even after 6 days of reaction time. These results provide further evidence that the proximity of the heteroatom influence the reactivity of the formyl group for the Baylis-Hillman reaction within a heterocyclic system. Similar to isoxazolecarbaldehydes, we speculate that the lone pair of the heteroatom might be instrumental in eliminating the DABCO from the intermediate. $^{5 b, c}$

It has been stated in the preceding text that within a heterocyclic system introduction of a halo group on a carbon adjacent to the carbon bearing the formyl group (as for pyridinecarbaldehydes) increases the reactivity of the formyl group for the Baylis-Hillman reaction. In our objective to evaluate whether the introduction of a halo-substituent on the carbon adjacent to the carbon containing the formyl has any positive effect on the reactivity of the formyl group in pyrazole-3-carbaldehyde, compounds 25a-c were prepared by treatment of 7a-c with iodine monochloride. ${ }^{13}$ The choice of introducing the iodo group was governed by the well documented synthetic utility of 4-iodo-pyrazoles for cross coupling protocols. ${ }^{14}$ Furthermore, the Baylis-Hillman reaction on this substrate would yield products suitable for generating pyrazoleannulated ring systems. It was pleasing to observe that the reaction of 25a-c with 1.5 equiv. of acrylonitrile in the presence of 0.5 equiv. of $\mathrm{DABCO}$ was complete at a better rate compared to other aldehydes (scheme 5).

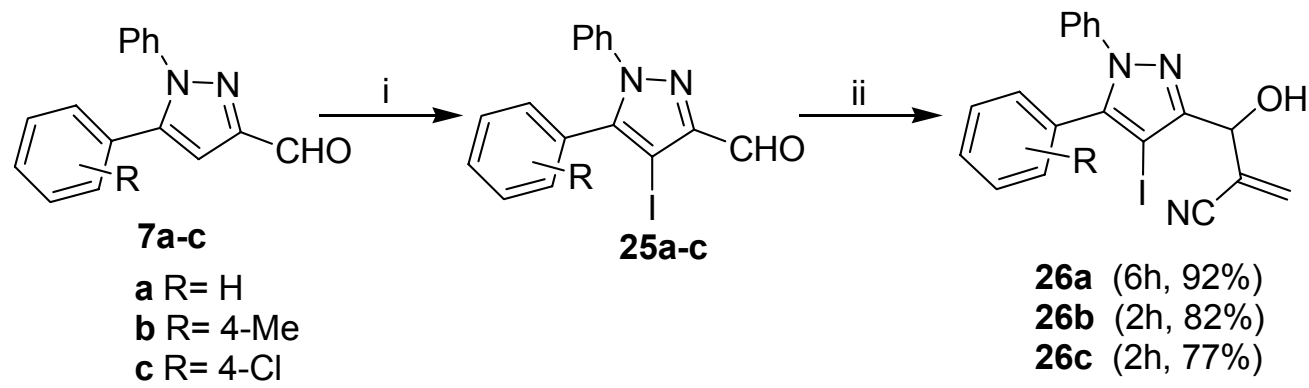

Scheme 5. Reagents and Conditions: i) $\mathrm{ICl}, \mathrm{K}_{2} \mathrm{CO}_{3}, \mathrm{CHCl}_{3}, \mathrm{rt}, 18 \mathrm{~h}$; ii) $\mathrm{CH}_{2} \mathrm{CH}=\mathrm{CN}$ (1.5 eq.), $\operatorname{DABCO}(0.5$ eq. $)$, rt.

\section{Conclusions}

In summary, our study provides further insight into the fact that within a heterocycle the formyl group present on the carbon atom adjacent to the heteroatom has better reactivity for the BaylisHillman reaction as compared to the formyl group which is present on the carbon away from the heteroatom. Several pyrazole derivatives synthesized during the present study may serve as useful starting material for generating new pyrazole derivatives including the pyrazole annulated scaffolds. The work towards this objective is underway in our laboratory. 


\section{Experimental Section}

Melting points are uncorrected and were determined in capillary tubes in apparatus containing silicon oil. IR spectra were recorded using a Perkin Elmer RX I FTIR spectrophotometer. ${ }^{1} \mathrm{H}$ NMR and ${ }^{13} \mathrm{C}$ NMR spectra were recorded on either a 300 or a $200 \mathrm{MHz}$ FT spectrometer, using TMS as an internal standard (chemical shifts in $\delta$ values, $J$ in Hz). The FABMS were recorded on JEOL/ SX-102 spectrometers and ESMS were recorded on a Micromass LCMS system. Elemental analyses were performed on a Carlo Erba 1108 microanalyzer or Elementar's Vario EL III microanalyzer.

General procedure for the Baylis-Hillman reaction- To a mixture of DABCO (for amount refer to Table 1,2 and 3) and appropriate alkene (for amount refer to Table 1,2 and 3) that has been stirred at r.t. for $20 \mathrm{~min}$. was added appropriate aldehyde $(5.3 \mathrm{mmol})$ under stirring and the reaction was allowed to proceed for a period as indicated in Table 1, 2 or 3 . Thereafter $5 \%$ aq. $\mathrm{HCl}$ soln. $(50 \mathrm{~mL})$ was added to the reaction mixture to neutralize the base and extracted with ethyl acetate $(2 \times 100 \mathrm{~mL})$. The organic layers were combined, washed with brine $(100 \mathrm{~mL})$, dried over anhyd. $\mathrm{Na}_{2} \mathrm{SO}_{4}$ and evaporated under vacuum to yield an oily residue. The residue was purified by column chromatography over silica gel (60-120 mesh) using hexane: ethyl acetate as eluent. A mixture of hexane: ethyl acetate $(85: 15, \mathrm{v} / \mathrm{v})$ yielded the starting material while further elution with 60:40 mixture furnished the desired products.

2-[(1,5-diphenyl-1H-pyrazol-3-yl)(hydroxy)methyl]acrylonitrile (9a). White solid, mp 119$120{ }^{\circ} \mathrm{C} ; v_{\max }(\mathrm{KBr}) 2224(\mathrm{CN}), 3259(\mathrm{OH}) \mathrm{cm}^{-1} ;{ }^{1} \mathrm{H} \mathrm{NMR}\left(\mathrm{CDCl}_{3}, 200 \mathrm{MHz}\right) \delta=3.36(\mathrm{~d}, 1 \mathrm{H}, J=$ $4.7 \mathrm{~Hz}, \mathrm{CHOH}), 5.49(\mathrm{~d}, 1 \mathrm{H}, J=4.2 \mathrm{~Hz}, \mathrm{CHOH}), 6.12\left(\mathrm{~s}, 1 \mathrm{H},=\mathrm{CH}_{2}\right), 6.25\left(\mathrm{~s}, 1 \mathrm{H},=\mathrm{CH}_{2}\right), 6.57$ $(\mathrm{s}, 1 \mathrm{H},=\mathrm{CH}), 7.21-7.33(\mathrm{~m}, 10 \mathrm{H}, \mathrm{ArH})$; mass $(\mathrm{ES}+) \mathrm{m} / z=302.2\left(\mathrm{M}^{+}+1\right)$. Anal Calcd. for $\mathrm{C}_{19} \mathrm{H}_{15} \mathrm{~N}_{3} \mathrm{O} ; \mathrm{C}, 75.73 ; \mathrm{H}, 5.02 ; \mathrm{N}, 13.94$. Found C, 75.50; H, 5.18; N, 13.99 .

2-\{Hydroxy [5-(4-methylphenyl)-1-phenyl-1H-pyrazol-3-yl]methyl\}acrylonitrile (9b). White solid, mp 118-119 ${ }^{\circ} \mathrm{C} ; v_{\max }(\mathrm{KBr}) 2228(\mathrm{CN}), 3241(\mathrm{OH}) \mathrm{cm}^{-1} ;{ }^{1} \mathrm{H}$ NMR $\left(\mathrm{CDCl}_{3}, 300 \mathrm{MHz}\right) \delta=$ $2.36\left(\mathrm{~s}, 3 \mathrm{H}, \mathrm{CH}_{3}\right), 3.35$ (brs, $\left.1 \mathrm{H}, \mathrm{CHOH}\right), 5.50(\mathrm{~s}, 1 \mathrm{H}, \mathrm{CHOH}), 6.13\left(\mathrm{~d}, 1 \mathrm{H}, J=1.2 \mathrm{~Hz},=\mathrm{CH}_{2}\right)$, $6.27\left(\mathrm{~d}, 1 \mathrm{H}, J=1.2 \mathrm{~Hz},=\mathrm{CH}_{2}\right), 6.55(\mathrm{~s}, 1 \mathrm{H},=\mathrm{CH}), 7.12(\mathrm{~s}, 4 \mathrm{H}, \mathrm{ArH}), 7.29-7.38(\mathrm{~m}, 5 \mathrm{H}, \mathrm{ArH})$; ${ }^{13} \mathrm{C}$ NMR $\left(\mathrm{CDCl}_{3}, 75 \mathrm{MHz}\right) \delta=21.7,69.4,105.4,117.5,125.6,125.8,127.3,128.2,129.0$, 129.4 129.6, 131.0, 139.0, 140.1, 145.4, 151.9; mass (ES+) $m / z=316.1\left(\mathrm{M}^{+}+1\right)$. Anal Calcd. for $\mathrm{C}_{20} \mathrm{H}_{17} \mathrm{~N}_{3} \mathrm{O} ; \mathrm{C}, 76.17 ; \mathrm{H}, 5.43 ; \mathrm{N}, 13.32$. Found C, 76.38; H, 5.22; N, 13.30.

2-[[5-(4-chlorophenyl)-1-phenyl-1H-pyrazol-3-yl](hydroxy)methyl]acrylonitrile (9c). White solid, mp 125-127 ${ }^{\circ} \mathrm{C} ; v_{\max }(\mathrm{KBr}) 2228(\mathrm{CN}), 3241(\mathrm{OH}) \mathrm{cm}^{-1} ;{ }^{1} \mathrm{H}$ NMR $\left(\mathrm{CDCl}_{3}, 300 \mathrm{MHz}\right) \delta=$ $3.18(\mathrm{~d}, 1 \mathrm{H}, J=5.1 \mathrm{~Hz}, \mathrm{CHOH}), 5.50(\mathrm{~d}, 1 \mathrm{H}, J=4.7 \mathrm{~Hz}, \mathrm{CHOH}), 6.14\left(\mathrm{~d}, 1 \mathrm{H}, J=1.3 \mathrm{~Hz},=\mathrm{CH}_{2}\right)$, $6.27\left(\mathrm{~d}, 1 \mathrm{H}, J=1.3 \mathrm{~Hz},=\mathrm{CH}_{2}\right), 6.59(\mathrm{~s}, 1 \mathrm{H},=\mathrm{CH}), 7.15-7.19(\mathrm{~m}, 2 \mathrm{H}, \mathrm{ArH}), 7.29-7.32(\mathrm{~m}, 4 \mathrm{H}$, $\mathrm{ArH}), 7.36-7.40(\mathrm{~m}, 3 \mathrm{H}, \mathrm{ArH}) ;{ }^{13} \mathrm{C} \mathrm{NMR}\left(\mathrm{CDCl}_{3}, 75 \mathrm{MHz}\right) \delta=69.4,105.8,117.4,125.4,125.7$, 128.6, 128.7, 129.3, 129.6, 130.4, 131.2, 135.2, 139.7, 144.0, 152.0; mass (ES+) $\mathrm{m} / \mathrm{z}=336.0$ $\left(\mathrm{M}^{+}+1\right)$. Anal Calcd. for $\mathrm{C}_{19} \mathrm{H}_{14} \mathrm{ClN}_{3} \mathrm{O} ; \mathrm{C}, 67.96 ; \mathrm{H}, 4.20 ; \mathrm{N}, 12.51$. Found $\mathrm{C}, 68.25 ; \mathrm{H}, 4.18 ; \mathrm{N}$, 12.66 . 
Methyl 2-[(1,5-diphenyl-1H-pyrazol-3-yl)(hydroxy)methyl]acrylate (10a). Yellow oil; $v_{\max }$ (Neat) $1720\left(\mathrm{CO}_{2} \mathrm{Me}\right), 3400(\mathrm{OH}) \mathrm{cm}^{-1} ;{ }^{1} \mathrm{H} \mathrm{NMR}\left(\mathrm{CDCl}_{3}, 300 \mathrm{MHz}\right) \delta=3.83\left(\mathrm{~s}, 3 \mathrm{H}, \mathrm{CO}_{2} \mathrm{CH}_{3}\right)$ $5.77(\mathrm{~s}, 1 \mathrm{H}, \mathrm{CHOH}), 6.08\left(\mathrm{~s}, 1 \mathrm{H},=\mathrm{CH}_{2}\right), 6.42\left(\mathrm{~s}, 1 \mathrm{H},=\mathrm{CH}_{2}\right), 6.51(\mathrm{~s}, 1 \mathrm{H},=\mathrm{CH}), 7.21-7.32(\mathrm{~m}$, $10 \mathrm{H}, \mathrm{ArH})$; mass $(\mathrm{ES}+) m / z=335.0\left(\mathrm{M}^{+}+1\right)$. Anal Calcd. for $\mathrm{C}_{20} \mathrm{H}_{18} \mathrm{~N}_{2} \mathrm{O}_{3} ; \mathrm{C}, 71.84 ; \mathrm{H}, 5.43 ; \mathrm{N}$, 8.38. Found C, 71.65; H, 5.48; N, 8.22.

Methyl 2-\{hydroxy[5-(4-methylphenyl)-1-phenyl-1 $H$-pyrazol-3-yl]methyl\}acrylate (10b). White solid, mp 119-121 ${ }^{\circ} \mathrm{C} ; v_{\max }(\mathrm{KBr}) 1720\left(\mathrm{CO}_{2} \mathrm{Me}\right), 3400(\mathrm{OH}) \mathrm{cm}^{-1} ;{ }^{1} \mathrm{H} \mathrm{NMR}\left(\mathrm{CDCl}_{3}, 300\right.$ $\mathrm{MHz}) \delta=2.35\left(\mathrm{~s}, 3 \mathrm{H}, \mathrm{CH}_{3}\right), 3.82\left(\mathrm{~s}, 3 \mathrm{H}, \mathrm{CO}_{2} \mathrm{CH}_{3}\right), 5.76(\mathrm{~s}, 1 \mathrm{H}, \mathrm{CHOH}), 6.07(\mathrm{~d}, 1 \mathrm{H}, J=0.9$, $\left.=\mathrm{CH}_{2}\right), 6.41\left(\mathrm{~s}, 1 \mathrm{H},=\mathrm{CH}_{2}\right), 6.47(\mathrm{~s}, 1 \mathrm{H},=\mathrm{CH}), 7.11(\mathrm{~s}, 4 \mathrm{H}, \mathrm{ArH}), 7.30-7.34(\mathrm{~m}, 5 \mathrm{H}, \operatorname{ArH}) ;{ }^{13} \mathrm{C}$ NMR $\left(\mathrm{CDCl}_{3}, 75 \mathrm{MHz}\right) \delta=21.4,52.1,68.6,105.6,125.4,126.6,127.6,128.7,129.0,129.3$, 138.4, 140.1, 144.3, 153.8, 167.0; mass (ES+) $m / z=349.1\left(\mathrm{M}^{+}+1\right)$. Anal Calcd. for $\mathrm{C}_{21} \mathrm{H}_{20} \mathrm{~N}_{2} \mathrm{O}_{3}$; C, 72.40; H, 5.79; N, 8.04. Found C, 72.51; H, 5.76; N, 7.85.

Methyl 2-[[5-(4-chlorophenyl)-1-phenyl-1H-pyrazol-3-yl](hydroxy)methyl]acrylate (10c). Yellow oil; $v_{\max }$ (Neat) $1721\left(\mathrm{CO}_{2} \mathrm{Me}\right), 3402(\mathrm{OH}) \mathrm{cm}^{-1 ; 1} \mathrm{H} \mathrm{NMR}\left(\mathrm{CDCl}_{3}, 300 \mathrm{MHz}\right) \delta=3.51(\mathrm{~d}$, $1 \mathrm{H}, J=6.3 \mathrm{~Hz}, \mathrm{CHOH}), 3.83\left(\mathrm{~s}, 3 \mathrm{H}, \mathrm{CO}_{2} \mathrm{CH}_{3}\right), 5.75$ (d, $\left.1 \mathrm{H}, J=5.4 \mathrm{~Hz}, \mathrm{CHOH}\right), 6.07$ (s, $1 \mathrm{H}$, $\left.=\mathrm{CH}_{2}\right), 6.42\left(\mathrm{~s}, 1 \mathrm{H},=\mathrm{CH}_{2}\right), 6.51(\mathrm{~s}, 1 \mathrm{H},=\mathrm{CH}), 7.14-7.17(\mathrm{~m}, 2 \mathrm{H}, \mathrm{ArH}), 7.27-7.38(\mathrm{~m}, 7 \mathrm{H}, \operatorname{ArH})$; ${ }^{13} \mathrm{C} \mathrm{NMR}\left(\mathrm{CDCl}_{3}, 75 \mathrm{MHz}\right) \delta=52.0,68.5,105.8,116.2,125.2,126.5,127.7,128.7,129.0$, $129.9,134.4,139.6,140.7,142.8,153.8,166.8$; mass $(\mathrm{ES}+) m / z=368.9\left(\mathrm{M}^{+}+1\right)$. Anal Calcd. for $\mathrm{C}_{20} \mathrm{H}_{17} \mathrm{ClN}_{2} \mathrm{O}_{3} ; \mathrm{C}, 65.13 ; \mathrm{H}, 4.65 ; \mathrm{N}, 7.60$. Found C, 65.21; H, 4.89; N, 7.61.

Ethyl 2-[(1,5-diphenyl-1H-pyrazol-3-yl)(hydroxy)methyl]acrylate (11a). Yellow oil; $v_{\max }$ (Neat) $1719\left(\mathrm{CO}_{2} \mathrm{Et}\right), 3292(\mathrm{OH}) \mathrm{cm}^{-1} ;{ }^{1} \mathrm{H} \mathrm{NMR}\left(\mathrm{CDCl}_{3}, 200 \mathrm{MHz}\right) \delta=1.30(\mathrm{t}, 3 \mathrm{H}, J=7.2 \mathrm{~Hz}$, $\mathrm{CH}_{3} \mathrm{CH}_{2}$ ), 3.70 (brs, $1 \mathrm{H}, \mathrm{CHOH}$ ), 4.26 (q, 2H, J=7.2 Hz, $\mathrm{CH}_{2} \mathrm{CH}_{3}$ ), 5.75 (s, 1H, CHOH), 6.04 $\left(\mathrm{s}, 1 \mathrm{H},=\mathrm{CH}_{2}\right), 6.40\left(\mathrm{~s}, 1 \mathrm{H},=\mathrm{CH}_{2}\right), 6.50(\mathrm{~s}, 1 \mathrm{H},=\mathrm{CH}), 7.22-7.29(\mathrm{~m}, 10 \mathrm{H}, \mathrm{ArH}) ;{ }^{13} \mathrm{C} \mathrm{NMR}$ $\left(\mathrm{CDCl}_{3}, 50 \mathrm{MHz}\right) \delta=14.6,61.4,69.0,106.2,125.7,126.7,127.9,128.7,128.9,129.1,129.3$, $130.8,140.3,141.5,144.4,154.2,166.9$; mass $(\mathrm{ES}+) m / z=349.1\left(\mathrm{M}^{+}+1\right)$. Anal Calcd. for $\mathrm{C}_{21} \mathrm{H}_{20} \mathrm{~N}_{2} \mathrm{O}_{3} ; \mathrm{C}, 72.40 ; \mathrm{H}, 5.79 ; \mathrm{N}, 8.04$. Found C, 72.33; H, 5.90; N, 7.88.

Ethyl 2-\{hydroxy[5-(4-methylphenyl)-1-phenyl-1 $H$-pyrazol-3-yl]methyl\}acrylate (11b). Yellow oil; $v_{\max }$ (Neat) $1716\left(\mathrm{CO}_{2} \mathrm{Et}\right), 3447(\mathrm{OH}) \mathrm{cm}^{-1} ;{ }^{1} \mathrm{H} \mathrm{NMR}\left(\mathrm{CDCl}_{3}, 300 \mathrm{MHz}\right) \delta=1.30(\mathrm{t}$, $\left.3 \mathrm{H}, J=7.2 \mathrm{~Hz}, \mathrm{CH}_{3} \mathrm{CH}_{2}\right), 2.06\left(\mathrm{~s}, 3 \mathrm{H}, \mathrm{CH}_{3}\right), 4.26\left(\mathrm{q}, 2 \mathrm{H}, J=7.2 \mathrm{~Hz}, \mathrm{CH}_{2} \mathrm{CH}_{3}\right), 5.75(\mathrm{~s}, 1 \mathrm{H}$, $\mathrm{CHOH}), 6.05\left(\mathrm{~s}, 1 \mathrm{H},=\mathrm{CH}_{2}\right), 6.41\left(\mathrm{~s}, 1 \mathrm{H},=\mathrm{CH}_{2}\right), 6.50(\mathrm{~s}, 1 \mathrm{H},=\mathrm{CH}), 7.12-7.15(\mathrm{~m}, 2 \mathrm{H}, \operatorname{ArH})$, 7.25-7.28 (m, 4H, ArH), 7.32-7.36 (m, 3H, ArH); ${ }^{13} \mathrm{C} \mathrm{NMR}\left(\mathrm{CDCl}_{3}, 50 \mathrm{MHz}\right) \delta=12.9,19.8$, 59.7, 67.2, 104.6, 124.1, 125.0, 126.5, 127.5, 127.8, 128.6, 133.1, 138.3, 139.7, 141.6, 152.7, 165.1; mass $(E S+) m / z=363.1\left(\mathrm{M}^{+}+1\right)$. Anal Calcd. for $\mathrm{C}_{22} \mathrm{H}_{22} \mathrm{~N}_{2} \mathrm{O}_{3} ; \mathrm{C}, 72.91 ; \mathrm{H}, 6.12 ; \mathrm{N}, 7.73$. Found C, 72.99; H, 6.13; N, 7.78.

Ethyl 2-[[5-(4-chlorophenyl)-1-phenyl-1H-pyrazol-3-yl](hydroxy)methyl]acrylate (11c). White solid, mp 128-130 ${ }^{\circ} \mathrm{C} ; v_{\max }(\mathrm{KBr}) 1714\left(\mathrm{CO}_{2} \mathrm{Et}\right), 3402(\mathrm{OH}) \mathrm{cm}^{-1} ;{ }^{1} \mathrm{H} \mathrm{NMR}\left(\mathrm{CDCl}_{3}, 200\right.$ $\mathrm{MHz}) \delta=1.29\left(\mathrm{t}, 3 \mathrm{H}, J=7.2 \mathrm{~Hz}, \mathrm{CH}_{3} \mathrm{CH}_{2}\right.$ ), 3.81 (brs, $\left.1 \mathrm{H}, \mathrm{CHOH}\right), 4.25$ (q, 2H, $J=7.2 \mathrm{~Hz}$, $\left.\mathrm{CH}_{2} \mathrm{CH}_{3}\right), 5.73(\mathrm{~s}, 1 \mathrm{H}, \mathrm{CHOH}), 6.04\left(\mathrm{~s}, 1 \mathrm{H},=\mathrm{CH}_{2}\right), 6.40\left(\mathrm{~s}, 1 \mathrm{H},=\mathrm{CH}_{2}\right), 6.49(\mathrm{~s}, 1 \mathrm{H},=\mathrm{CH}), 7.13$ $(\mathrm{d}, 2 \mathrm{H}, J=8.6 \mathrm{~Hz}, \mathrm{ArH}), 7.24-7.37$ (m, 7H, $\mathrm{ArH}) ;{ }^{13} \mathrm{C} \mathrm{NMR}\left(\mathrm{CDCl}_{3}, 50 \mathrm{MHz}\right) \delta=14.6,61.4$, $69.0,106.3,125.7,126.7,127.4,128.2,129.2,129.5,130.3,134.8,140.0,141.4,143.2,154.4$, 
166.8; mass (ES+) $m / z=383.0\left(\mathrm{M}^{+}+1\right)$. Anal Calcd. for $\mathrm{C}_{21} \mathrm{H}_{19} \mathrm{ClN}_{2} \mathrm{O}_{3} ; \mathrm{C}, 65.88 ; \mathrm{H}, 5.00 ; \mathrm{N}$, 7.32. Found C, 65.67; H, 5.11; N, 7.36.

tert-Butyl 2-[(1,5-diphenyl-1H-pyrazol-3-yl)(hydroxy)methyl]acrylate (12a). White solid; mp

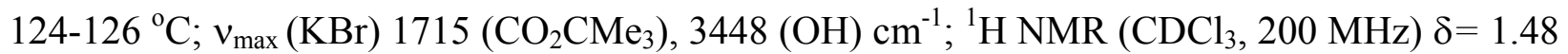
(s, 9H, $\left.\mathrm{CMe}_{3}\right), 3.70(\mathrm{~d}, 1 \mathrm{H}, J=6.7 \mathrm{~Hz}, \mathrm{CHOH}), 5.72(\mathrm{~d}, 1 \mathrm{H}, J=6.7 \mathrm{~Hz}, \mathrm{CHOH}), 5.93(\mathrm{~s}, 1 \mathrm{H}$, $\left.=\mathrm{CH}_{2}\right), 6.30\left(\mathrm{~s}, 1 \mathrm{H},=\mathrm{CH}_{2}\right), 6.50(\mathrm{~s}, 1 \mathrm{H},=\mathrm{CH}), 7.21-7.31(\mathrm{~s}, 10 \mathrm{H}, \mathrm{ArH}) ;{ }^{13} \mathrm{C} \mathrm{NMR}\left(\mathrm{CDCl}_{3}, 50\right.$ $\mathrm{MHz}) \delta=28.5,69.3,82.0,106.1,125.7,125.9,127.8,128.7,128.9,129.1,129.3,130.9,140.4$, 142.7, 144.4, 154.5, 166.2; mass (ES+) $m / z=377.0\left(\mathrm{M}^{+}+1\right)$. Anal Calcd. for $\mathrm{C}_{23} \mathrm{H}_{24} \mathrm{~N}_{2} \mathrm{O}_{3} \mathrm{C}$, 73.38; H, 6.43; N, 7.44. Found C, 73.21; H, 6.45; N, 7.59.

tert-Butyl 2-\{hydroxy[5-(4-methylphenyl)-1-phenyl-1 H-pyrazol-3-yl]methyl\}acrylate (12b). White solid, mp 116-118 ${ }^{\circ} \mathrm{C} ; v_{\max }(\mathrm{KBr}) 1710\left(\mathrm{CO}_{2} \mathrm{CMe}_{3}\right), 3436(\mathrm{OH}) \mathrm{cm}^{-1} ;{ }^{1} \mathrm{H} \mathrm{NMR}\left(\mathrm{CDCl}_{3}\right.$, $300 \mathrm{MHz}) \delta=1.50\left(\mathrm{~s}, 9 \mathrm{H}, \mathrm{CMe}_{3}\right), 2.35\left(\mathrm{~s}, 3 \mathrm{H}, \mathrm{CH}_{3}\right), 3.57(\mathrm{~d}, 1 \mathrm{H}, J=6.6 \mathrm{~Hz}, \mathrm{CHOH}), 5.69$ (d, $1 \mathrm{H}, J=6.1 \mathrm{~Hz}, \mathrm{CHOH}), 5.94\left(\mathrm{~s}, 1 \mathrm{H},=\mathrm{CH}_{2}\right), 6.31\left(\mathrm{~s}, 1 \mathrm{H},=\mathrm{CH}_{2}\right), 6.48(\mathrm{~s}, 1 \mathrm{H},=\mathrm{CH}), 7.12(\mathrm{~s}, 4 \mathrm{H}$, ArH)), 7.29-7.36 (m, 5H, ArH); mass (ES+) $m / z=391.0\left(\mathrm{M}^{+}+1\right)$. Anal Calcd. for $\mathrm{C}_{24} \mathrm{H}_{26} \mathrm{~N}_{2} \mathrm{O}_{3}$; C, 73.82; H, 6.71; N, 7.17. Found C, 73.81; H, 6.76; N, 7.28.

tert-Butyl 2-[[5-(4-chlorophenyl)-1-phenyl-1H-pyrazol-3-yl](hydroxy)methyl]acrylate (12c). White solid, mp $123-125{ }^{\circ} \mathrm{C} ; v_{\max }(\mathrm{KBr}) 1710\left(\mathrm{CO}_{2} \mathrm{CMe}_{3}\right), 3418(\mathrm{OH}) \mathrm{cm}^{-1} ;{ }^{1} \mathrm{H} \mathrm{NMR}\left(\mathrm{CDCl}_{3}\right.$, $200 \mathrm{MHz}) \delta=1.48\left(\mathrm{~s}, 9 \mathrm{H}, \mathrm{CMe}_{3}\right), 3.69(\mathrm{~d}, 1 \mathrm{H}, J=6.6 \mathrm{~Hz}, \mathrm{CHOH}), 5.67$ (d, 1H, $J=6.6 \mathrm{~Hz}$, $\mathrm{CHOH}), 5.93\left(\mathrm{~s}, 1 \mathrm{H},=\mathrm{CH}_{2}\right), 6.30\left(\mathrm{~s}, 1 \mathrm{H},=\mathrm{CH}_{2}\right), 6.50(\mathrm{~s}, 1 \mathrm{H},=\mathrm{CH}), 7.13(\mathrm{~d}, 2 \mathrm{H}, J=8.2 \mathrm{~Hz}$, $\mathrm{ArH})$ ), 7.24-7.32 (m, 7H, ArH); ${ }^{13} \mathrm{C} \mathrm{NMR}\left(\mathrm{CDCl}_{3}, 50 \mathrm{MHz}\right) \delta=28.5,69.3,82.0,106.2,125.7$, $126.0,128.1,129.2,129.4,130.3,134.8,140.1,142.5,143.2,154.6,166.2$; mass $(\mathrm{ES}+) \mathrm{m} / z=$ $410.9\left(\mathrm{M}^{+}+1\right)$. Anal Calcd. for $\mathrm{C}_{23} \mathrm{H}_{23} \mathrm{ClN}_{2} \mathrm{O}_{3} ; \mathrm{C}, 67.23 ; \mathrm{H}, 5.64 ; \mathrm{N}, 6.82$. Found $\mathrm{C}, 67.14 ; \mathrm{H}$, $5.69 ; \mathrm{N}, 6.90$.

2-[(1,5-diphenyl-1H-pyrazol-3-yl)(hydroxy)methyl]cyclohex-2-en-1-one (13a). White solid,

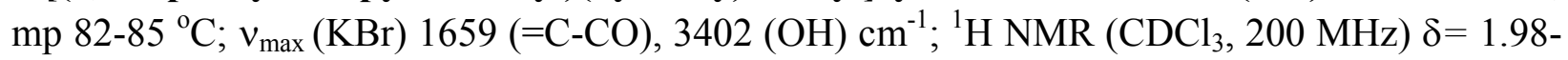
$2.17\left(\mathrm{~m}, 2 \mathrm{H}, \mathrm{CH}_{2}\right), 2.41-2.55\left(\mathrm{~m}, 4 \mathrm{H}, 2 \mathrm{xCH}_{2}\right), 3.90$ (brs, 1H, $\left.\mathrm{CHOH}\right), 5.70$ (s, 1H, CHOH), 6.52 $(\mathrm{s}, 1 \mathrm{H},=\mathrm{CH}), 7.05(\mathrm{t}, 1 \mathrm{H}, J=3.8 \mathrm{~Hz},=\mathrm{CH}), 7.19(\mathrm{~m}, 10 \mathrm{H}, \mathrm{ArH}) ;{ }^{13} \mathrm{C} \mathrm{NMR}\left(\mathrm{CDCl}_{3}, 50 \mathrm{MHz}\right) \delta=$ 22.9, 26.2, 39.0, 68.8, 106.4, 125.7, 127.8, 128.7, 128.8, 129.1, 129.3, 130.8, 140.1, 140.4, 144.4, 148.0, 154.5, 200.9; mass (ES+) $m / z=345.1\left(\mathrm{M}^{+}+1\right)$. Anal Calcd. for $\mathrm{C}_{22} \mathrm{H}_{20} \mathrm{~N}_{2} \mathrm{O}_{2} ; \mathrm{C}, 76.72 ; \mathrm{H}$, $5.85 ; \mathrm{N}, 8.13$. Found C, 76.65; H, 5.64; N, 8.31.

2-\{Hydroxy [5-(4-methylphenyl)-1-phenyl-1 H-pyrazol-3-yl]methyl\}cyclohex-2-en-1-one (13b). Yellow oil; $v_{\max }$ (Neat) $1668(=\mathrm{C}-\mathrm{CO}), 3402(\mathrm{OH}) \mathrm{cm}^{-1} ;{ }^{1} \mathrm{H} \mathrm{NMR}\left(\mathrm{CDCl}_{3}, 200 \mathrm{MHz}\right) \delta=$ 2.00-2.10 (m, 2H, $\left.\mathrm{CH}_{2}\right), 2.33\left(\mathrm{~s}, 3 \mathrm{H}, \mathrm{CH}_{3}\right), 2.41-2.54\left(\mathrm{~m}, 4 \mathrm{H}, 2 \mathrm{xCH}_{2}\right), 3.91$ (brs, $\left.1 \mathrm{H}, \mathrm{CHOH}\right)$, $5.69(\mathrm{~s}, 1 \mathrm{H}, \mathrm{CHOH}), 6.48(\mathrm{~s}, 1 \mathrm{H},=\mathrm{CH}), 7.03-7.09(\mathrm{~m}, 5 \mathrm{H},=\mathrm{CH}$ and $\mathrm{ArH}), 7.30-7.32(\mathrm{~s}, 5 \mathrm{H}$, ArH); mass $(\mathrm{ES}+) m / z=359.0\left(\mathrm{M}^{+}+1\right)$. Anal Calcd. for $\mathrm{C}_{23} \mathrm{H}_{22} \mathrm{~N}_{2} \mathrm{O}_{2} ; \mathrm{C}, 77.07 ; \mathrm{H}, 6.19 ; \mathrm{N}, 7.82$. Found C, 76.92; H, 6.33; N, 7.87.

2-[[5-(4-Chlorophenyl)-1-phenyl-1H-pyrazol-3-yl](hydroxy)methyl]cyclohex-2-en-1-one (13c). White solid, mp 149-151 ${ }^{\circ} \mathrm{C} ; v_{\max }(\mathrm{KBr}) 1668$ (=C-CO), $3402(\mathrm{OH}) \mathrm{cm}^{-1}$; ${ }^{1} \mathrm{H}$ NMR $\left(\mathrm{CDCl}_{3}, 200 \mathrm{MHz}\right) \delta=1.97-2.09\left(\mathrm{~m}, 2 \mathrm{H}, \mathrm{CH}_{2}\right), 2.40-2.54\left(\mathrm{~m}, 4 \mathrm{H}, 2 \mathrm{xCH}_{2}\right), 3.96$ (brs, 1H, $\mathrm{CHOH}), 5.68(\mathrm{~s}, 1 \mathrm{H}, \mathrm{CHOH}), 6.52(\mathrm{~s}, 1 \mathrm{H},=\mathrm{CH}), 7.05(\mathrm{t}, 1 \mathrm{H}, J=3.8 \mathrm{~Hz},=\mathrm{CH}), 7.11-7.35(\mathrm{~m}$, 
$9 \mathrm{H}, \mathrm{ArH}) ;{ }^{13} \mathrm{C} \mathrm{NMR}\left(\mathrm{CDCl}_{3}, 50 \mathrm{MHz}\right) \delta=22.9,26.2,39.0,68.9,106.5,125.7,128.1,129.1$, 129.3, 129.4, 130.3, 134.7, 140.1, 143.1, 148.1, 154.7, 200.9; mass (ES+) $m / z=379.0\left(\mathrm{M}^{+}+1\right)$. Anal Calcd. for $\mathrm{C}_{22} \mathrm{H}_{19} \mathrm{ClN}_{2} \mathrm{O}_{2} ; \mathrm{C}, 69.75 ; \mathrm{H}, 5.05 ; \mathrm{N}, 7.39$. Found $\mathrm{C}, 69.77 ; \mathrm{H}, 4.95 ; \mathrm{N}, 7.50$.

2-[(1,3-Diphenyl-1H-pyrazol-5-yl)(hydroxy)methyl]acrylonitrile (14a). White solid, mp 155$157{ }^{\circ} \mathrm{C} ; v_{\max }(\mathrm{KBr}) 2228(\mathrm{CN}), 3404(\mathrm{OH}) \mathrm{cm}^{-1} ;{ }^{1} \mathrm{H} \mathrm{NMR}\left(\mathrm{CDCl}_{3}, 300 \mathrm{MHz}\right) \delta=2.80(\mathrm{~d}, 1 \mathrm{H}, J=$ $5.7 \mathrm{~Hz}, \mathrm{CHOH}), 5.34(\mathrm{~d}, 1 \mathrm{H}, J=3.8 \mathrm{~Hz}, \mathrm{CHOH}), 6.11\left(\mathrm{~d}, 2 \mathrm{H}, J=0.6 \mathrm{~Hz},=\mathrm{CH}_{2}\right), 6.83(\mathrm{~s}, 1 \mathrm{H}$, $=\mathrm{CH})$, 7.36-7.48 (m, 8H, ArH), $7.85(\mathrm{~d}, 2 \mathrm{H}, J=8.0 \mathrm{~Hz}, \mathrm{ArH})$; mass $(\mathrm{ES}+) \mathrm{m} / z=302.3\left(\mathrm{M}^{+}+1\right)$. Anal Calcd. for $\mathrm{C}_{19} \mathrm{H}_{15} \mathrm{~N}_{3} \mathrm{O} ; \mathrm{C}, 75.73 ; \mathrm{H}, 5.02 ; \mathrm{N}, 13.94$. Found 75.77; H, 4.88; N, 14.02 .

2-\{Hydroxy [3-(4-methylphenyl)-1-phenyl-1H-pyrazol-5-yl]methyl\}acrylonitrile

(14b). White solid, mp 133-135 ${ }^{\circ} \mathrm{C} ; v_{\max }(\mathrm{KBr}) 2227(\mathrm{CN}), 3218(\mathrm{OH}) \mathrm{cm}^{-1} ;{ }^{1} \mathrm{H} \mathrm{NMR}\left(\mathrm{CDCl}_{3}, 300\right.$ $\mathrm{MHz}) \delta=2.40\left(\mathrm{~s}, 3 \mathrm{H}, \mathrm{CH}_{3}\right), 2.95(\mathrm{~d}, 1 \mathrm{H}, J=5.0 \mathrm{~Hz}, \mathrm{CHOH}), 5.30(\mathrm{~s}, 1 \mathrm{H}, \mathrm{CHOH}), 6.07(\mathrm{~s}, 1 \mathrm{H}$, $\left.=\mathrm{CH}_{2}\right), 6.08\left(\mathrm{~d}, 1 \mathrm{H}, J=1.5 \mathrm{~Hz},=\mathrm{CH}_{2}\right), 6.77(\mathrm{~s}, 1 \mathrm{H},=\mathrm{CH}), 7.23-7.28(\mathrm{~m}, 3 \mathrm{H}, \mathrm{ArH}), 7.46-7.52(\mathrm{~m}$, $4 \mathrm{H}, \mathrm{ArH}), 7.86(\mathrm{~d}, 2 \mathrm{H}, J=8.0 \mathrm{~Hz}, \mathrm{ArH}) ;{ }^{13} \mathrm{C} \mathrm{NMR}\left(\mathrm{CDCl}_{3}, 50 \mathrm{MHz}\right) \delta=21.7,66.0,104.2$, $117.0,124.3,126.1,126.2,129.2,129.8,131.3,138.7,139.2,142.8,152.6$; mass $(\mathrm{ES}+) \mathrm{m} / \mathrm{z}=$ $316.3\left(\mathrm{M}^{+}+1\right)$. Anal Calcd. for $\mathrm{C}_{20} \mathrm{H}_{17} \mathrm{~N}_{3} \mathrm{O} ; \mathrm{C}, 76.17 ; \mathrm{H}, 5.43 ; \mathrm{N}, 13.32$. Found 76.28; H, 5.44; $\mathrm{N}$, 13.20 .

2-[[3-(4-Chlorophenyl)-1-phenyl-1H-pyrazol-5-yl](hydroxy)methyl]acrylonitrile

(14c). White solid, mp 142-144 ${ }^{\circ} \mathrm{C} ; v_{\max }(\mathrm{KBr}) 2230(\mathrm{CN}), 3332(\mathrm{OH}) \mathrm{cm}^{-1} ;{ }^{1} \mathrm{H} \mathrm{NMR}\left(\mathrm{CDCl}_{3}, 300\right.$ $\mathrm{MHz}) \delta=2.60$ (brs, $1 \mathrm{H}, \mathrm{CHOH}), 5.37(\mathrm{~s}, 1 \mathrm{H}, \mathrm{CHOH}), 6.12\left(\mathrm{~s}, 1 \mathrm{H},=\mathrm{CH}_{2}\right), 6.13(\mathrm{~d}, 1 \mathrm{H}, J=1.1$ $\left.\mathrm{Hz},=\mathrm{CH}_{2}\right), 6.82(\mathrm{~s}, 1 \mathrm{H},=\mathrm{CH}), 7.38-7.61(\mathrm{~m}, 7 \mathrm{H}, \mathrm{ArH}), 7.81(\mathrm{~d}, 2 \mathrm{H}, J=8.4 \mathrm{~Hz}, \mathrm{ArH}) ;{ }^{13} \mathrm{C} \mathrm{NMR}$ $\left(\mathrm{CDCl}_{3}, 50 \mathrm{MHz}\right) \delta=66.0,104.3,116.9,124.2,126.1,127.5,129.3,129.5,129.9,131.2,131.5$, 134.6, 139.0, 143.1, 151.4; mass $(\mathrm{ES}+) \mathrm{m} / \mathrm{z}=336.2\left(\mathrm{M}^{+}+1\right)$. Anal Calcd. for $\mathrm{C}_{19} \mathrm{H}_{14} \mathrm{ClN}_{3} \mathrm{O}$; $\mathrm{C}$, 67.96; H, 4.20; N, 12.51. Found C, 68.11; H, 4.27; N, 12.44.

Methyl 2-[(1,3-diphenyl-1H-pyrazol-5-yl)(hydroxy)methyl]acrylate (15a)- White solid, mp 99-101 ${ }^{\circ} \mathrm{C} ; v_{\max }(\mathrm{KBr}) 1720\left(\mathrm{CO}_{2} \mathrm{Me}\right), 3411(\mathrm{OH}) \mathrm{cm}^{-1} ;{ }^{1} \mathrm{H} \mathrm{NMR}\left(\mathrm{CDCl}_{3}, 300 \mathrm{MHz}\right) \delta=2.95(\mathrm{~d}$, $1 \mathrm{H}, J=4.8 \mathrm{~Hz}, \mathrm{CHOH}), 3.74\left(\mathrm{~s}, 3 \mathrm{H}, \mathrm{CO}_{2} \mathrm{CH}_{3}\right), 5.64(\mathrm{~d}, 1 \mathrm{H}, J=3.8 \mathrm{~Hz}, \mathrm{CHOH}), 5.99(\mathrm{~s}, 1 \mathrm{H}$, $\left.=\mathrm{CH}_{2}\right), 6.42\left(\mathrm{~s}, 1 \mathrm{H},=\mathrm{CH}_{2}\right), 6.67(\mathrm{~s}, 1 \mathrm{H},=\mathrm{CH}), 7.33-7.67(\mathrm{~m}, 8 \mathrm{H}, \mathrm{ArH}), 7.82-7.90(\mathrm{~m}, 2 \mathrm{H}, \mathrm{ArH})$; mass $(\mathrm{ES}+) m / z=335.2\left(\mathrm{M}^{+}+1\right)$. Anal Calcd. for $\mathrm{C}_{20} \mathrm{H}_{18} \mathrm{~N}_{2} \mathrm{O}_{3} ; \mathrm{C}, 71.84 ; \mathrm{H}, 5.43 ; \mathrm{N}, 8.38$. Found C, 71.90; H, 5.33; N, 8.38.

Methyl 2-\{hydroxy[3-(4-methylphenyl)-1-phenyl-1 $H$-pyrazol-5-yl]methyl\}acrylate (15b). White solid, mp 105-108 ${ }^{\circ} \mathrm{C} ; v_{\max }(\mathrm{KBr}) 1720\left(\mathrm{CO}_{2} \mathrm{Me}\right), 3407(\mathrm{OH}) \mathrm{cm}^{-1} ;{ }^{1} \mathrm{H} \mathrm{NMR}\left(\mathrm{CDCl}_{3}, 300\right.$ $\mathrm{MHz}) \delta=2.39\left(\mathrm{~s}, 3 \mathrm{H}, \mathrm{CH}_{3}\right), 2.95(\mathrm{brs}, 1 \mathrm{H}, \mathrm{CHOH}), 3.74\left(\mathrm{~s}, 3 \mathrm{H}, \mathrm{CO}_{2} \mathrm{CH}_{3}\right), 5.64(\mathrm{~s}, 1 \mathrm{H}, \mathrm{CHOH})$, $5.99\left(\mathrm{~s}, 1 \mathrm{H},=\mathrm{CH}_{2}\right), 6.41\left(\mathrm{~s}, 1 \mathrm{H},=\mathrm{CH}_{2}\right), 6.64(\mathrm{~s}, 1 \mathrm{H},=\mathrm{CH}), 7.22(\mathrm{~d}, 2 \mathrm{H}, J=8.0 \mathrm{~Hz}, \mathrm{ArH}), 7.44-$ $7.54(\mathrm{~m}, 3 \mathrm{H}, \mathrm{ArH}), 7.65$ (d, 2H, $J=8.0 \mathrm{~Hz}, \mathrm{ArH}), 7.76(\mathrm{~d}, 2 \mathrm{H}, J=8.0 \mathrm{~Hz}, \mathrm{ArH})$; mass $(\mathrm{ES}+) \mathrm{m} / z=$ $349.3\left(\mathrm{M}^{+}+1\right)$. Anal Calcd. for $\mathrm{C}_{21} \mathrm{H}_{20} \mathrm{~N}_{2} \mathrm{O}_{3} ; \mathrm{C}, 72.40 ; \mathrm{H}, 5.79 ; \mathrm{N}, 8.04$. Found C, 72.68; H, 5.55; N, 7.91 .

Methyl 2-[[3-(4-chlorophenyl)-1-phenyl-1H-pyrazol-5-yl](hydroxy)methyl]acrylate (15c). Yellow oil; $v_{\max }$ (Neat) $1719\left(\mathrm{CO}_{2} \mathrm{Me}\right), 3336(\mathrm{OH}) \mathrm{cm}^{-1} ;{ }^{1} \mathrm{H} \mathrm{NMR}\left(\mathrm{CDCl}_{3}, 300 \mathrm{MHz}\right) \delta=2.88$ $(\mathrm{d}, 1 \mathrm{H}, J=5.2 \mathrm{~Hz}, \mathrm{CHOH}), 3.75\left(\mathrm{~s}, 3 \mathrm{H}, \mathrm{CO}_{2} \mathrm{CH}_{3}\right), 5.64(\mathrm{~d}, 1 \mathrm{H}, J=2.3 \mathrm{~Hz}, \mathrm{CHOH}), 5.98(\mathrm{~s}, 1 \mathrm{H}$, $\left.=\mathrm{CH}_{2}\right), 6.42\left(\mathrm{~s}, 1 \mathrm{H},=\mathrm{CH}_{2}\right), 6.64(\mathrm{~s}, 1 \mathrm{H},=\mathrm{CH}), 7.36-7.40(\mathrm{~m}, 2 \mathrm{H}, \mathrm{ArH}), 7.44-7.56(\mathrm{~m}, 5 \mathrm{H}, \mathrm{ArH})$, 
$7.80(\mathrm{~d}, 2 \mathrm{H}, J=8.0 \mathrm{~Hz}, \mathrm{ArH}) ;{ }^{13} \mathrm{C} \mathrm{NMR}\left(\mathrm{CDCl}_{3}, 75 \mathrm{MHz}\right) \delta=52.3,65.0,103.3,125.8,126.3$, 127.2, 128.7, 128.9, 129.5, 131.6, 133.9, 139.5, 140.5, 145.1, 150.7, 166.3; mass (ES+) $\mathrm{m} / \mathrm{z}=$ 369.2 $\left(\mathrm{M}^{+}+1\right)$. Anal Calcd. for $\mathrm{C}_{20} \mathrm{H}_{17} \mathrm{ClN}_{2} \mathrm{O}_{3} ; \mathrm{C}, 65.13 ; \mathrm{H}, 4.65 ; \mathrm{N}, 7.60$. Found $\mathrm{C}, 65.01 ; \mathrm{H}$, $4.88 ; \mathrm{N}, 7.51$.

Ethyl 2-[(1,3-diphenyl-1H-pyrazol-5-yl)(hydroxy)methyl]acrylate (16a). White solid, mp $118-120{ }^{\circ} \mathrm{C} ; v_{\max }(\mathrm{KBr}) 1713\left(\mathrm{CO}_{2} \mathrm{Et}\right), 3421(\mathrm{OH}) \mathrm{cm}^{-1} ;{ }^{1} \mathrm{H} \mathrm{NMR}\left(\mathrm{CDCl}_{3}, 200 \mathrm{MHz}\right) \delta=1.21(\mathrm{t}$, $\left.3 \mathrm{H}, J=7.1 \mathrm{~Hz}, \mathrm{CH}_{3} \mathrm{CH}_{2}\right), 2.91(\mathrm{~d}, 1 \mathrm{H}, J=6.7 \mathrm{~Hz}, \mathrm{CHOH}), 4.17$ (q, $2 \mathrm{H}, J=7.1 \mathrm{~Hz}, \mathrm{CH}_{2} \mathrm{CH}_{3}$ ), $5.62(\mathrm{~d}, 1 \mathrm{H}, J=6.7 \mathrm{~Hz}, \mathrm{CHOH}), 5.96\left(\mathrm{~s}, 1 \mathrm{H},=\mathrm{CH}_{2}\right), 6.40\left(\mathrm{~s}, 1 \mathrm{H},=\mathrm{CH}_{2}\right), 6.65(\mathrm{~s}, 1 \mathrm{H},=\mathrm{CH})$, 7.31-7.51 (m, 6H, ArH), 7.63-7.67 (dd, $\left.2 \mathrm{H}, J_{1}=1.2 \mathrm{~Hz}, J_{2}=8.0 \mathrm{~Hz}, \mathrm{ArH}\right), 7.85(\mathrm{~d}, 2 \mathrm{H}, J=8.0$ $\mathrm{Hz}, \mathrm{ArH}) ;{ }^{13} \mathrm{C} \mathrm{NMR}\left(\mathrm{CDCl}_{3}, 50 \mathrm{MHz}\right) \delta=14.5,61.5,65.2,103.6,125.9,126.2,128.4,128.7$, 129.0, 129.7, 133.3, 139.8, 141.1, 145.1, 152.0, 166.1; mass (ES+) $m / z=349.2\left(\mathrm{M}^{+}+1\right)$. Anal Calcd. for $\mathrm{C}_{21} \mathrm{H}_{20} \mathrm{~N}_{2} \mathrm{O}_{3} ; \mathrm{C}, 72.40 ; \mathrm{H}, 5.79 ; \mathrm{N}, 8.04$. Found C, 72.22; H, 5.85; N, 7.84.

Ethyl 2-\{hydroxy[3-(4-methylphenyl)-1-phenyl-1 H-pyrazol-5-yl]methyl\}acrylate (16b). Yellow oil; $v_{\max }$ (Neat) $1713\left(\mathrm{CO}_{2} \mathrm{Et}\right), 3381(\mathrm{OH}) \mathrm{cm}^{-1} ;{ }^{1} \mathrm{H} \mathrm{NMR}\left(\mathrm{CDCl}_{3}, 200 \mathrm{MHz}\right) \delta=1.21(\mathrm{t}$, $\left.3 \mathrm{H}, J=7.2 \mathrm{~Hz}, \mathrm{CH}_{3} \mathrm{CH}_{2}\right), 2.40\left(\mathrm{~s}, 3 \mathrm{H}, \mathrm{CH}_{3}\right), 2.90(\mathrm{~d}, 1 \mathrm{H}, J=6.7 \mathrm{~Hz}, \mathrm{CHOH}), 4.16$ (q, 2H, $J=7.2$ $\left.\mathrm{Hz}, \mathrm{CH}_{2} \mathrm{CH}_{3}\right), 5.62(\mathrm{~d}, 1 \mathrm{H}, J=6.3 \mathrm{~Hz}, \mathrm{CHOH}), 5.96\left(\mathrm{~s}, 1 \mathrm{H},=\mathrm{CH}_{2}\right), 6.40\left(\mathrm{~s}, 1 \mathrm{H},=\mathrm{CH}_{2}\right), 6.62(\mathrm{~s}$, $1 \mathrm{H},=\mathrm{CH}), 7.20(\mathrm{~d}, 2 \mathrm{H}, J=8.0 \mathrm{~Hz}, \mathrm{ArH}), 7.44-7.54(\mathrm{~m}, 3 \mathrm{H}, \mathrm{ArH}), 7.62-7.67(\mathrm{~m}, 2 \mathrm{H}, \mathrm{ArH}), 7.73$ (d, $2 \mathrm{H}, J=8.0 \mathrm{~Hz}, \mathrm{ArH})$; mass $(\mathrm{ES}+) \mathrm{m} / z=363.3\left(\mathrm{M}^{+}+1\right)$. Anal Calcd. for $\mathrm{C}_{22} \mathrm{H}_{22} \mathrm{~N}_{2} \mathrm{O}_{3} \cdot \mathrm{H}_{2} \mathrm{O} ; \mathrm{C}$, 69.46; H, 6.36; N, 7.36. Found C, 69.28; H, 6.35; N, 7.15.

Ethyl 2-[[3-(4-chlorophenyl)-1-phenyl-1H-pyrazol-5-yl](hydroxy)methyl]acrylate (16c). Yellow oil; $v_{\max }(\mathrm{Neat}) 1718\left(\mathrm{CO}_{2} \mathrm{Et}\right), 3356(\mathrm{OH}) \mathrm{cm}^{-1} ;{ }^{1} \mathrm{H} \mathrm{NMR}\left(\mathrm{CDCl}_{3}, 200 \mathrm{MHz}\right) \delta=1.19(\mathrm{t}$, $\left.3 \mathrm{H}, J=7.2 \mathrm{~Hz}, \mathrm{CH}_{3} \mathrm{CH}_{2}\right), 3.12(\mathrm{~d}, 1 \mathrm{H}, J=5.3 \mathrm{~Hz}, \mathrm{CHOH}), 4.14\left(\mathrm{q}, 2 \mathrm{H}, J=7.2 \mathrm{~Hz}, \mathrm{CH}_{2} \mathrm{CH}_{3}\right)$, $5.57(\mathrm{~d}, 1 \mathrm{H}, J=5.4 \mathrm{~Hz}, \mathrm{CHOH}), 5.93\left(\mathrm{~s}, 1 \mathrm{H},=\mathrm{CH}_{2}\right), 6.38\left(\mathrm{~s}, 1 \mathrm{H},=\mathrm{CH}_{2}\right), 6.58(\mathrm{~s}, 1 \mathrm{H},=\mathrm{CH}), 7.34$ $(\mathrm{d}, 2 \mathrm{H}, J=8.0 \mathrm{~Hz}, \mathrm{ArH}), 7.42-7.51(\mathrm{~m}, 3 \mathrm{H}, \mathrm{ArH}), 7.59-7.63\left(\mathrm{dd}, 2 \mathrm{H}, J_{1}=1.4 \mathrm{~Hz}, J_{2}=8.0 \mathrm{~Hz}\right.$, ArH), $7.75(\mathrm{~d}, 2 \mathrm{H}, J=8.0 \mathrm{~Hz}, \mathrm{ArH}) ;{ }^{13} \mathrm{C}$ NMR $\left(\mathrm{CDCl}_{3}, 50 \mathrm{MHz}\right) \delta=14.5,61.6,65.3,103.5$, 125.9, 126.3, 127.4, 128.9, 129.2, 129.7, 131.8, 134.1, 139.7, 140.9, 145.3, 150.9, 166.1; mass (ES+) $m / z=383.2\left(\mathrm{M}^{+}+1\right)$. Anal Calcd. for $\mathrm{C}_{21} \mathrm{H}_{19} \mathrm{ClN}_{2} \mathrm{O}_{3} ; \mathrm{C}, 65.88 ; \mathrm{H}, 5.00 ; \mathrm{N}, 7.32$. Found $\mathrm{C}$, $65.74 ; \mathrm{H}, 5.20 ; \mathrm{N}, 7.45$.

tert-Butyl Ethyl 2-[(1,3-diphenyl-1H-pyrazol-5-yl)(hydroxy)methyl]acrylate (17a). White solid, mp 167-169 ${ }^{\circ} \mathrm{C} ; v_{\max }(\mathrm{KBr}) 1718\left(\mathrm{CO}_{2} \mathrm{CMe}_{3}\right), 3422(\mathrm{OH}) \mathrm{cm}^{-1} ;{ }^{1} \mathrm{H} \mathrm{NMR}\left(\mathrm{CDCl}_{3}, 300\right.$ $\mathrm{MHz}) \delta=1.40\left(\mathrm{~s}, 9 \mathrm{H}, \mathrm{CMe}_{3}\right), 2.97(\mathrm{~d}, 1 \mathrm{H}, J=7.1 \mathrm{~Hz}, \mathrm{CHOH}), 5.58(\mathrm{~d}, 1 \mathrm{H}, J=7.1 \mathrm{~Hz}, \mathrm{CHOH})$, $5.88\left(\mathrm{~s}, 1 \mathrm{H},=\mathrm{CH}_{2}\right), 6.33\left(\mathrm{~s}, 1 \mathrm{H},=\mathrm{CH}_{2}\right), 6.67(\mathrm{~s}, 1 \mathrm{H},=\mathrm{CH}), 7.33-7.51(\mathrm{~m}, 6 \mathrm{H}, \mathrm{ArH}), 7.69(\mathrm{~d}, 2 \mathrm{H}$, $J=8.0 \mathrm{~Hz}, \mathrm{ArH}), 7.87(\mathrm{~d}, 2 \mathrm{H}, J=8.0 \mathrm{~Hz}, \mathrm{ArH}) ;{ }^{13} \mathrm{C} \mathrm{NMR}\left(\mathrm{CDCl}_{3}, 50 \mathrm{MHz}\right) \delta=28.4,65.4,82.3$, 103.7, 125.5, 125.7, 126.2, 128.4, 128.6, 129.0, 129.6, 133.3, 139.9, 142.3, 145.3, 152.0, 165.4; mass (ES+) $m / z=377.1\left(\mathrm{M}^{+}+1\right)$. Anal Calcd. for $\mathrm{C}_{23} \mathrm{H}_{24} \mathrm{~N}_{2} \mathrm{O}_{3} ; \mathrm{C}, 73.38 ; \mathrm{H}, 6.43 ; \mathrm{N}, 7.44$. Found C, 73.51; H, 6.44; N, 7.31.

tert-Butyl 2-\{hydroxy[3-(4-methylphenyl)-1-phenyl-1 $H$-pyrazol-5-yl]methyl\}acrylate (17b). Yellow oil; $v_{\max }$ (Neat) $1719\left(\mathrm{CO}_{2} \mathrm{CMe}_{3}\right), 3425(\mathrm{OH}) \mathrm{cm}^{-1} ;{ }^{1} \mathrm{H}$ NMR $\left(\mathrm{CDCl}_{3}, 300 \mathrm{MHz}\right) \delta=1.40$ (s, 9H, $\mathrm{CMe}_{3}$ ), 2.39 (s, 3H, $\mathrm{CH}_{3}$ ), $2.95(\mathrm{~d}, 1 \mathrm{H}, J=7.1 \mathrm{~Hz}, \mathrm{CHOH}), 5.57(\mathrm{~d}, 1 \mathrm{H}, J=7.1 \mathrm{~Hz}$, $\mathrm{CHOH}), 5.88\left(\mathrm{~s}, 1 \mathrm{H},=\mathrm{CH}_{2}\right), 6.33\left(\mathrm{~s}, 1 \mathrm{H},=\mathrm{CH}_{2}\right), 6.63(\mathrm{~s}, 1 \mathrm{H},=\mathrm{CH}), 7.26(\mathrm{~d}, 2 \mathrm{H}, J=8.0 \mathrm{~Hz}$, 
ArH), 7.43-7.54 ( m, 3H, ArH), 7.66-7.77 (m, 4H, ArH); mass (ES+) $m /=391.1\left(\mathrm{M}^{+}+1\right)$. Anal Calcd. for $\mathrm{C}_{24} \mathrm{H}_{26} \mathrm{~N}_{2} \mathrm{O}_{3} . \mathrm{H}_{2} \mathrm{O} ; \mathrm{C}, 70.57 ; \mathrm{H}, 6.91 ; \mathrm{N}, 6.86$. Found C, 70.57; H, 6.91; N, 6.86.

tert-Butyl 2-[[3-(4-chlorophenyl)-1-phenyl-1H-pyrazol-5-yl](hydroxy)methyl]acrylate (17c). Yellow oil; $v_{\max }$ (Neat) $1705\left(\mathrm{CO}_{2} \mathrm{CMe}_{3}\right), 3444(\mathrm{OH}) \mathrm{cm}^{-1} ;{ }^{1} \mathrm{H} \mathrm{NMR}\left(\mathrm{CDCl}_{3}, 200 \mathrm{MHz}\right) \delta=1.36$ $\left(\mathrm{s}, 9 \mathrm{H}, \mathrm{CMe}_{3}\right), 3.16$ (brs, $\left.1 \mathrm{H}, \mathrm{CHOH}\right), 5.51(\mathrm{~s}, 1 \mathrm{H}, \mathrm{CHOH}), 5.85\left(\mathrm{~s}, 1 \mathrm{H},=\mathrm{CH}_{2}\right), 6.30(\mathrm{~s}, 1 \mathrm{H}$, $\left.=\mathrm{CH}_{2}\right), 6.59(\mathrm{~s}, 1 \mathrm{H},=\mathrm{CH}), 7.33-7.49(\mathrm{~m}, 5 \mathrm{H}, \mathrm{ArH}), 7.63-7.67\left(\mathrm{dd}, 2 \mathrm{H}, J_{1}=1.2 \mathrm{~Hz}, J_{2}=8.2 \mathrm{~Hz}\right.$, $\operatorname{ArH}), 7.76(\mathrm{~d}, 2 \mathrm{H}, J=8.4 \mathrm{~Hz}, \mathrm{ArH}) ;{ }^{13} \mathrm{C} \mathrm{NMR}\left(\mathrm{CDCl}_{3}, 50 \mathrm{MHz}\right) \delta=28.4,65.5,82.4,103.6$, $125.7,127.4,128.8,129.2,129.7,131.9,134.1,139.7,142.2,145.5,150.9$, 165.4; mass (ES+) $m / z=411.0\left(\mathrm{M}^{+}+1\right)$. Anal Calcd. for $\mathrm{C}_{23} \mathrm{H}_{23} \mathrm{ClN}_{2} \mathrm{O}_{3} ; \mathrm{C}, 67.23 ; \mathrm{H}, 5.64 ; \mathrm{N}, 6.82$. Found $\mathrm{C}, 67.01$; $\mathrm{H}, 5.78 ; \mathrm{N}, 6.71$.

3-[(1,3-Diphenyl-1H-pyrazol-5-yl)(hydroxy)methyl]but-3-en-2-one (18a). Yellow oil; $v_{\max }$ (Neat) $1665(=\mathrm{C}-\mathrm{CO}), 3401(\mathrm{OH}) \mathrm{cm}^{-1} ;{ }^{1} \mathrm{H} \mathrm{NMR}\left(\mathrm{CDCl}_{3}, 200 \mathrm{MHz}\right) \delta=2.35\left(\mathrm{~s}, 3 \mathrm{H}, \mathrm{CH}_{3}\right), 3.01$ $(\mathrm{d}, 1 \mathrm{H}, J=6.0 \mathrm{~Hz}, \mathrm{CHOH}), 5.68(\mathrm{~d}, 1 \mathrm{H}, J=5.4 \mathrm{~Hz}, \mathrm{CHOH}), 6.10\left(\mathrm{~s}, 1 \mathrm{H},=\mathrm{CH}_{2}\right), 6.23(\mathrm{~s}, 1 \mathrm{H}$, $\left.=\mathrm{CH}_{2}\right), 6.62(\mathrm{~s}, 1 \mathrm{H},=\mathrm{CH}), 7.30-7.54(\mathrm{~m}, 6 \mathrm{H}, \mathrm{ArH}), 7.62(\mathrm{~d}, 2 \mathrm{H}, J=7.8 \mathrm{~Hz}, \mathrm{ArH}), 7.84(\mathrm{~d}, 2 \mathrm{H}$, $J=7.8 \mathrm{~Hz}$, ArH); mass (ES+) $m / z=319.3\left(\mathrm{M}^{+}+1\right)$. Anal Calcd. for $\mathrm{C}_{20} \mathrm{H}_{18} \mathrm{~N}_{2} \mathrm{O}_{2} ; \mathrm{C}, 75.45 ; \mathrm{H}$, 5.70; N, 8.80. Found C, 75.34; H, 5.73; N, 8.86.

3-\{Hydroxy[3-(4-methylphenyl)-1-phenyl-1H-pyrazol-5-yl]methyl\}but-3-en-2-one (18b). Yellow oil; $v_{\max }$ (Neat) $1675(=\mathrm{C}-\mathrm{CO}), 3383(\mathrm{OH}) \mathrm{cm}^{-1} ;{ }^{1} \mathrm{H} \mathrm{NMR}\left(\mathrm{CDCl}_{3}, 200 \mathrm{MHz}\right) \delta=2.35(\mathrm{~s}$, $\left.3 \mathrm{H}, \mathrm{CH}_{3}\right), 2.37\left(\mathrm{~s}, 3 \mathrm{H}, \mathrm{CH}_{3}\right), 2.98(\mathrm{~d}, 1 \mathrm{H}, J=4.4 \mathrm{~Hz}, \mathrm{CHOH}), 5.67(\mathrm{~d}, 1 \mathrm{H}, J=4.4 \mathrm{~Hz}, \mathrm{CHOH})$, $6.10\left(\mathrm{~s}, 1 \mathrm{H},=\mathrm{CH}_{2}\right), 6.24\left(\mathrm{~s}, 1 \mathrm{H},=\mathrm{CH}_{2}\right), 6.59(\mathrm{~s}, 1 \mathrm{H},=\mathrm{CH}), 7.20(\mathrm{~d}, 2 \mathrm{H}, J=8.0 \mathrm{~Hz}, \mathrm{ArH}), 7.44-$ $7.53(\mathrm{~m}, 3 \mathrm{H}, \mathrm{ArH}), 7.62\left(\mathrm{dd}, 2 \mathrm{H}, J_{1}=1.7 \mathrm{~Hz}, J_{2}=8.0 \mathrm{~Hz}, \operatorname{ArH}\right), 7.73$ (d, 2H, J=8.0 Hz, ArH); mass (ES+) $m / z=333.3\left(\mathrm{M}^{+}+1\right), 334.3\left(\mathrm{M}^{+}+2\right)$. Anal Calcd. for $\mathrm{C}_{21} \mathrm{H}_{20} \mathrm{~N}_{2} \mathrm{O}_{2} ; \mathrm{C}, 75.88 ; \mathrm{H}, 6.06$; N, 8.43. Found C, 75.95; H, 6.14; N, 8.59.

3-[[3-(4-Chlorophenyl)-1-phenyl-1H-pyrazol-5-yl](hydroxy)methyl]but-3-en-2-one (18c). Yellow oil; $v_{\max }(\mathrm{Neat}) 1665(=\mathrm{C}-\mathrm{CO}), 3339(\mathrm{OH}) \mathrm{cm}^{-1} ;{ }^{1} \mathrm{H} \mathrm{NMR}\left(\mathrm{CDCl}_{3}, 300 \mathrm{MHz}\right) \delta=2.38(\mathrm{~s}$, $\left.3 \mathrm{H}, \mathrm{CH}_{3}\right), 5.69(\mathrm{~s}, 1 \mathrm{H}, \mathrm{CHOH}), 6.13\left(\mathrm{~s}, 1 \mathrm{H},=\mathrm{CH}_{2}\right), 6.26\left(\mathrm{~s}, 1 \mathrm{H},=\mathrm{CH}_{2}\right), 6.61(\mathrm{~s}, 1 \mathrm{H},=\mathrm{CH}), 7.38$ (d, 2H, $J=8.4 \mathrm{~Hz}, \mathrm{ArH}), 7.45-7.55$ (m, 3H, ArH), 7.63 (d, 2H, $J=8.4 \mathrm{~Hz}, \mathrm{ArH}), 7.79$ (d, 2H, $J=$ $8.4 \mathrm{~Hz}, \mathrm{ArH})$; mass $(\mathrm{ES}+) \mathrm{m} / z=353.3\left(\mathrm{M}^{+}+1\right), 355.2\left(\mathrm{M}^{+}+3\right)$. Anal Calcd. for $\mathrm{C}_{20} \mathrm{H}_{17} \mathrm{ClN}_{2} \mathrm{O}_{2}$; $\mathrm{C}$, 68.09; H, 4.86; N, 7.94. Found C, 67.88; H, 4.77; N, 8.09.

2-[(1,3-Diphenyl-1H-pyrazol-5-yl)(hydroxy)methyl]cyclohex-2-en-1-one (19a). Yellow oil; $v_{\max }$ (Neat) $1668(=\mathrm{C}-\mathrm{CO}), 3402(\mathrm{OH}) \mathrm{cm}^{-1} ;{ }^{1} \mathrm{H} \mathrm{NMR}\left(\mathrm{CDCl}_{3}, 300 \mathrm{MHz}\right) \delta=1.96-2.01(\mathrm{~m}, 2 \mathrm{H}$, $\mathrm{CH}_{2}$ ), 2.35-2.40 (m, 2H, $\left.\mathrm{CH}_{2}\right), 2.43-2.49\left(\mathrm{~m}, 2 \mathrm{H}, \mathrm{CH}_{2}\right), 3.47$ (brs, $\left.1 \mathrm{H}, \mathrm{CHOH}\right), 5.63(\mathrm{~s}, 1 \mathrm{H}$, $\mathrm{CHOH}), 6.73(\mathrm{~s}, 1 \mathrm{H},=\mathrm{CH}), 6.82(\mathrm{t}, 1 \mathrm{H}, J=4.5 \mathrm{~Hz},=\mathrm{CH}), 7.30-7.70(\mathrm{~m}, 8 \mathrm{H}, \mathrm{ArH}), 7.88(\mathrm{~d}, 2 \mathrm{H}$, $J=8.4 \mathrm{~Hz}, \mathrm{ArH})$; mass $(\mathrm{ES}+) m / z=379.8\left(\mathrm{M}^{+}+1\right)$. Anal Calcd. for $\mathrm{C}_{22} \mathrm{H}_{20} \mathrm{~N}_{2} \mathrm{O}_{2} . \mathrm{H}_{2} \mathrm{O} ; \mathrm{C}, 72.91 ; \mathrm{H}$, 6.12; N, 7.73. Found C, 73.07; H, 5.92; N, 7.60.

\section{2-\{Hydroxy[3-(4-methylphenyl)-1-phenyl-1H-pyrazol-5-yl]methyl\} cyclohex-2-en-1-one}

(19b). White solid, mp 130-133 ${ }^{\circ} \mathrm{C}$; $v_{\max }(\mathrm{KBr}) 1668$ (=C-CO), $3418(\mathrm{OH}) \mathrm{cm}^{-1}$; ${ }^{1} \mathrm{H} \mathrm{NMR}$ $\left(\mathrm{CDCl}_{3}, 300 \mathrm{MHz}\right) \delta=1.94-2.02\left(\mathrm{~m}, 2 \mathrm{H}, \mathrm{CH}_{2}\right), 2.35-2.39\left(\mathrm{~m}, 5 \mathrm{H}, \mathrm{CH}_{2}\right.$ and $\left.\mathrm{CH}_{3}\right), 2.43-2.49(\mathrm{~m}$, $\left.2 \mathrm{H}, \mathrm{CH}_{2}\right), 5.62(\mathrm{~s}, 1 \mathrm{H}, \mathrm{CHOH}), 6.69(\mathrm{~s}, 1 \mathrm{H},=\mathrm{CH}), 6.81(\mathrm{t}, 1 \mathrm{H}, J=4.0 \mathrm{~Hz},=\mathrm{CH}), 7.22(\mathrm{~d}, 2 \mathrm{H}, J=$ 8.0 Hz, ArH), 7.33-7.52 (m, 3H, ArH), 7.56-7.60 (m, 2H, ArH), 7.76 (d, 2H, J=8.0 Hz, ArH); 
mass $(\mathrm{ES}+) m / z=359.3\left(\mathrm{M}^{+}+1\right), 360.3\left(\mathrm{M}^{+}+2\right)$. Anal Calcd. for $\mathrm{C}_{23} \mathrm{H}_{22} \mathrm{~N}_{2} \mathrm{O}_{2} . \mathrm{H}_{2} \mathrm{O} ; \mathrm{C}, 73.38 ; \mathrm{H}$, $6.43 ; \mathrm{N}, 7.44$. Found C, 73.58; H, 6.29; N, 7.53.

2-[[3-(4-Chlorophenyl)-1-phenyl-1H-pyrazol-5-yl](hydroxy)methyl]cyclohex-2-en-1-one (19c). Yellow oil; $v_{\max }(\mathrm{Neat}) 1668(=\mathrm{C}-\mathrm{CO}), 3377(\mathrm{OH}) \mathrm{cm}^{-1} ;{ }^{1} \mathrm{H}$ NMR $\left(\mathrm{CDCl}_{3}, 200 \mathrm{MHz}\right) \delta=$ 1.88-2.00 (m, 2H, $\left.\mathrm{CH}_{2}\right), 2.30-2.45\left(\mathrm{~m}, 4 \mathrm{H}, 2 \mathrm{xCH}_{2}\right), 3.67$ (brs, $\left.1 \mathrm{H}, \mathrm{CHOH}\right), 5.56(\mathrm{~s}, 1 \mathrm{H}, \mathrm{CHOH})$, $6.65(\mathrm{~s}, 1 \mathrm{H},=\mathrm{CH}), 6.79(\mathrm{t}, 1 \mathrm{H}, J=3.9 \mathrm{~Hz},=\mathrm{CH}), 7.33-7.56(\mathrm{~m}, 7 \mathrm{H}, \mathrm{ArH}), 7.77(\mathrm{~d}, 2 \mathrm{H}, J=8.5$ $\mathrm{Hz}, \mathrm{ArH}) ;{ }^{13} \mathrm{C} \mathrm{NMR}\left(\mathrm{CDCl}_{3}, 50 \mathrm{MHz}\right) \delta=22.8,26.1,38.8,65.6,103.7,126.1,127.4,128.9$, 129.2 , 129.7, 131.9, 134.0, 138.9, 139.9, 145.9, 148.4, 150.9, 200.2; mass (ES+) $m / z=379.3$ $\left(\mathrm{M}^{+}+1\right), 381.2\left(\mathrm{M}^{+}+3\right)$. Anal Calcd. for $\mathrm{C}_{22} \mathrm{H}_{19} \mathrm{ClN}_{2} \mathrm{O}_{2} . \mathrm{H}_{2} \mathrm{O} ; \mathrm{C}, 66.58 ; \mathrm{H}, 5.33 ; \mathrm{N}, 7.06$. Found $\mathrm{C}$, $66.40 ; \mathrm{H}, 5.21 ; \mathrm{N}, 6.87$.

2-[(1,3-Diphenyl-1H-pyrazol-4-yl)(hydroxy)methyl]acrylonitrile (21a). White solid, mp 114$116{ }^{\circ} \mathrm{C} ; v_{\max }(\mathrm{KBr}) 2226(\mathrm{CN}), 3437(\mathrm{OH}) \mathrm{cm}^{-1} ;{ }^{1} \mathrm{H} \mathrm{NMR}\left(\mathrm{CDCl}_{3}, 300 \mathrm{MHz}\right) \delta=2.47(\mathrm{~d}, 1 \mathrm{H}, J=$ $4.7 \mathrm{~Hz}, \mathrm{CHOH}), 5.51(\mathrm{~d}, 1 \mathrm{H}, J=3.5 \mathrm{~Hz}, \mathrm{CHOH}), 6.11\left(\mathrm{~d}, 1 \mathrm{H}, J=1.1 \mathrm{~Hz},=\mathrm{CH}_{2}\right), 6.16(\mathrm{~d}, 1 \mathrm{H}, J=$ $\left.1.1 \mathrm{~Hz},=\mathrm{CH}_{2}\right), 7.32-7.37(\mathrm{~m}, 1 \mathrm{H}, \mathrm{ArH}), 7.44-7.52(\mathrm{~m}, 5 \mathrm{H}, \mathrm{ArH}), 7.73-7.79(\mathrm{~m}, 4 \mathrm{H}, \mathrm{ArH}), 8.09$ $(\mathrm{s}, 1 \mathrm{H},=\mathrm{CH})$; mass $(\mathrm{ES}+) \mathrm{m} / z=302.3\left(\mathrm{M}^{+}+1\right)$. Anal Calcd. for $\mathrm{C}_{19} \mathrm{H}_{15} \mathrm{~N}_{3} \mathrm{O} ; \mathrm{C}, 75.73 ; \mathrm{H}, 5.02 ; \mathrm{N}$, 13.94. Found C, 75.78; H, 5.11; N, 13.68 .

2-\{Hydroxy[3-(4-methylphenyl)-1-phenyl-1H-pyrazol-4-yl]methyl\}acrylonitrile

(21b). White solid, mp 140-142 ${ }^{\circ} \mathrm{C} ; v_{\max }(\mathrm{KBr}) 2226(\mathrm{CN}), 3266(\mathrm{OH}) \mathrm{cm}^{-1} ;{ }^{1} \mathrm{H} \mathrm{NMR}\left(\mathrm{CDCl}_{3}, 200\right.$ $\mathrm{MHz}) \delta=2.41\left(\mathrm{~s}, 3 \mathrm{H}, \mathrm{CH}_{3}\right), 2.80\left(\right.$ brs, $1 \mathrm{H}, \mathrm{CHOH}$, replaceable by $\left.\mathrm{D}_{2} \mathrm{O}\right), 5.47(\mathrm{~s}, 1 \mathrm{H}, \mathrm{CHOH})$, $6.07\left(\mathrm{~d}, 1 \mathrm{H}, J=1.1 \mathrm{~Hz},=\mathrm{CH}_{2}\right), 6.13\left(\mathrm{~d}, 1 \mathrm{H}, J=1.4 \mathrm{~Hz},=\mathrm{CH}_{2}\right), 7.24-7.50(\mathrm{~m}, 5 \mathrm{H}, \mathrm{ArH}), 7.62(\mathrm{~d}$, $2 \mathrm{H}, J=8.0 \mathrm{~Hz}, \mathrm{ArH}), 7.73(\mathrm{~d}, 2 \mathrm{H}, J=8.0 \mathrm{~Hz}, \mathrm{ArH}), 8.07(\mathrm{~s}, 1 \mathrm{H},=\mathrm{CH}) ;{ }^{13} \mathrm{C} \mathrm{NMR}\left(\mathrm{CDCl}_{3}, 50\right.$ $\mathrm{MHz}) \delta=21.7,66.7,117.6,119.7,120.5,126.0,127.3,127.4,128.6,129.8,129.9,130.8,138.9$, 140.0, 152.1; mass (ES+) $m / z=330.3\left(\mathrm{M}^{+}+1\right)$. Anal Calcd. for $\mathrm{C}_{20} \mathrm{H}_{17} \mathrm{~N}_{3} \mathrm{O} ; \mathrm{C}, 76.17 ; \mathrm{H}, 5.43 ; \mathrm{N}$, 13.32. Found C, 76.02; H, 5.48; N, 13.29.

2-[[3-(4-Chlorophenyl)-1-phenyl-1H-pyrazol-4-yl](hydroxy)methyl]acrylonitrile

(21c). White solid, mp 97-99 ${ }^{\circ} \mathrm{C} ; v_{\max }(\mathrm{KBr}) 2227(\mathrm{CN}), 3483(\mathrm{OH}) \mathrm{cm}^{-1} ;{ }^{1} \mathrm{H} \mathrm{NMR}\left(\mathrm{CDCl}_{3}, 200 \mathrm{MHz}\right)$ $\delta=2.56(\mathrm{brs}, 1 \mathrm{H}, \mathrm{CHOH}), 5.44(\mathrm{~s}, 1 \mathrm{H}, \mathrm{CHOH}), 6.09\left(\mathrm{~d}, 1 \mathrm{H}, J=1.2 \mathrm{~Hz},=\mathrm{CH}_{2}\right), 6.15(\mathrm{~d}, 1 \mathrm{H}, J=$ $\left.1.2 \mathrm{~Hz},=\mathrm{CH}_{2}\right), 7.29-7.51(\mathrm{~m}, 5 \mathrm{H}, \mathrm{ArH}), 7.65-7.74(\mathrm{~m}, 4 \mathrm{H}, \mathrm{ArH}), 8.05(\mathrm{~s}, 1 \mathrm{H},=\mathrm{CH})$; mass $(\mathrm{ES}+)$ $m / z=336.3\left(\mathrm{M}^{+}+1\right), 338.3\left(\mathrm{M}^{+}+3\right)$. Anal Calcd. for $\mathrm{C}_{19} \mathrm{H}_{14} \mathrm{ClN}_{3} \mathrm{O} ; \mathrm{C}, 67.96 ; \mathrm{H}, 4.20 ; \mathrm{N}, 12.51$. Found C, 68.13; H, 4.37; N, 12.52 .

Methyl 2-[(1,3-diphenyl-1H-pyrazol-4-yl)(hydroxy)methyl]acrylate (22a)- Yellow oil; $v_{\max }$ (Neat) $1718\left(\mathrm{CO}_{2} \mathrm{Me}\right), 3418(\mathrm{OH}) \mathrm{cm}^{-1} ;{ }^{1} \mathrm{H} \mathrm{NMR}\left(\mathrm{CDCl}_{3}, 200 \mathrm{MHz}\right) \delta=3.01$ (brs, $\left.1 \mathrm{H}, \mathrm{CHOH}\right)$, $3.77\left(\mathrm{~s}, 3 \mathrm{H}, \mathrm{CO}_{2} \mathrm{CH}_{3}\right), 5.81(\mathrm{~s}, 1 \mathrm{H}, \mathrm{CHOH}), 5.91\left(\mathrm{~s}, 1 \mathrm{H},=\mathrm{CH}_{2}\right), 6.36\left(\mathrm{~s}, 1 \mathrm{H},=\mathrm{CH}_{2}\right), 7.38-7.49$ (m, 5H, ArH), 7.73-7.79 (m, 5H, ArH), $7.97(\mathrm{~s}, 1 \mathrm{H},=\mathrm{CH})$; mass $(\mathrm{ES}+) m / z=335.2\left(\mathrm{M}^{+}+1\right)$. Anal Calcd. for $\mathrm{C}_{20} \mathrm{H}_{18} \mathrm{~N}_{2} \mathrm{O}_{3} ; \mathrm{C}, 71.84 ; \mathrm{H}, 5.43 ; \mathrm{N}, 8.38$. Found C, 72.09; H, 5.49; N, 8.45.

Methyl 2-\{hydroxy[3-(4-methylphenyl)-1-phenyl-1 $H$-pyrazol-4-yl]methyl\}acrylate (22b).

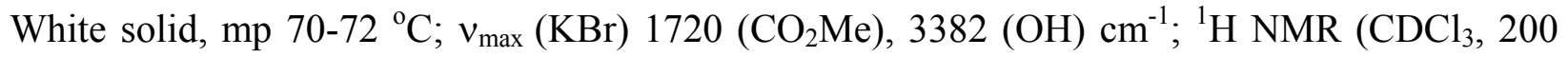
$\mathrm{MHz}) \delta=2.40\left(\mathrm{~s}, 3 \mathrm{H}, \mathrm{CH}_{3}\right), 2.80(\mathrm{brs}, 1 \mathrm{H}, \mathrm{CHOH}), 3.77\left(\mathrm{~s}, 3 \mathrm{H}, \mathrm{CO}_{2} \mathrm{CH}_{3}\right), 5.80(\mathrm{~s}, 1 \mathrm{H}, \mathrm{CHOH})$, $5.90\left(\mathrm{~s}, 1 \mathrm{H},=\mathrm{CH}_{2}\right), 6.35\left(\mathrm{~s}, 1 \mathrm{H},=\mathrm{CH}_{2}\right), 7.23-7.31(\mathrm{~m}, 3 \mathrm{H}, \mathrm{ArH}), 7.41-7.49(\mathrm{~m}, 2 \mathrm{H}, \mathrm{ArH}), 7.66$ $(\mathrm{d}, 2 \mathrm{H}, J=8.0 \mathrm{~Hz}, \operatorname{ArH}), 7.74(\mathrm{~d}, 2 \mathrm{H}, J=8.0 \mathrm{~Hz}, \mathrm{ArH}), 7.95(\mathrm{~s}, 1 \mathrm{H},=\mathrm{CH})$; mass $(\mathrm{ES}+) \mathrm{m} / z=$ 
349.2 $\left(\mathrm{M}^{+}+1\right)$. Anal Calcd. for $\mathrm{C}_{21} \mathrm{H}_{20} \mathrm{~N}_{2} \mathrm{O}_{3} ; \mathrm{C}, 72.40 ; \mathrm{H}, 5.79 ; \mathrm{N}, 8.04$. Found C, 72.32; H, 5.53; N, 8.28.

Methyl 2-[[3-(4-chlorophenyl)-1-phenyl-1H-pyrazol-4-yl](hydroxy)methyl]acrylate (22c). White solid, mp 128-130 ${ }^{\circ} \mathrm{C} ; v_{\max }(\mathrm{KBr}) 1719\left(\mathrm{CO}_{2} \mathrm{Me}\right), 3422(\mathrm{OH}) \mathrm{cm}^{-1} ;{ }^{1} \mathrm{H} \mathrm{NMR}\left(\mathrm{CDCl}_{3}, 200\right.$ $\mathrm{MHz}) \delta=2.95(\mathrm{brs}, 1 \mathrm{H}, \mathrm{CHOH}), 3.79\left(\mathrm{~s}, 3 \mathrm{H}, \mathrm{CO}_{2} \mathrm{CH}_{3}\right), 5.78(\mathrm{~s}, 1 \mathrm{H}, \mathrm{CHOH}), 5.92(\mathrm{~s}, 1 \mathrm{H}$, $\left.=\mathrm{CH}_{2}\right), 6.38\left(\mathrm{~s}, 1 \mathrm{H},=\mathrm{CH}_{2}\right), 7.29-7.34(\mathrm{~m}, 1 \mathrm{H}, \mathrm{ArH}), 7.42-7.50(\mathrm{~m}, 4 \mathrm{H}, \mathrm{ArH}), 7.72-7.80(\mathrm{~m}, 4 \mathrm{H}$, $\mathrm{ArH}), 7.95(\mathrm{~s}, 1 \mathrm{H},=\mathrm{CH})$; mass $(\mathrm{ES}+) \mathrm{m} / z=369.2\left(\mathrm{M}^{+}+1\right)$. Anal Calcd. for $\mathrm{C}_{20} \mathrm{H}_{17} \mathrm{ClN}_{2} \mathrm{O}_{3} ; \mathrm{C}$, $65.13 ; \mathrm{H}, 4.65 ; \mathrm{N}, 7.60$. Found C, 64.88; H, 4.50; N, 7.71.

Ethyl 2-\{hydroxy[3-(4-methylphenyl)-1-phenyl-1H-pyrazol-4-yl]methyl\}acrylate (23b). Yellow oil; $v_{\max }$ (Neat) $1712\left(\mathrm{CO}_{2} \mathrm{Et}\right), 3428(\mathrm{OH}) \mathrm{cm}^{-1} ;{ }^{1} \mathrm{H} \mathrm{NMR}\left(\mathrm{CDCl}_{3}, 200 \mathrm{MHz}\right) \delta=1.26(\mathrm{t}$, $\left.3 \mathrm{H}, J=7.2 \mathrm{~Hz}, \mathrm{CH}_{3} \mathrm{CH}_{2}\right), 2.40\left(\mathrm{~s}, 3 \mathrm{H}, \mathrm{CH}_{3}\right), 4.22\left(\mathrm{q}, 2 \mathrm{H}, J=7.2 \mathrm{~Hz}, \mathrm{CH}_{2} \mathrm{CH}_{3}\right), 5.79(\mathrm{~s}, 1 \mathrm{H}$, $\mathrm{CHOH}), 5.88\left(\mathrm{~s}, 1 \mathrm{H},=\mathrm{CH}_{2}\right), 6.34\left(\mathrm{~s}, 1 \mathrm{H},=\mathrm{CH}_{2}\right), 7.23-7.36(\mathrm{~m}, 3 \mathrm{H}, \mathrm{ArH}), 7.40-7.48(\mathrm{~m}, 2 \mathrm{H}$, $\operatorname{ArH}), 7.66(\mathrm{~d}, 2 \mathrm{H}, J=8.0 \mathrm{~Hz}, \operatorname{ArH}), 7.73(\mathrm{~d}, 2 \mathrm{H}, J=8.0 \mathrm{~Hz}, \operatorname{ArH}), 7.95(\mathrm{~s}, 1 \mathrm{H},=\mathrm{CH})$; mass $(\mathrm{ES}+) \mathrm{m} / z=363.2\left(\mathrm{M}^{+}+1\right)$. Anal Calcd. for $\mathrm{C}_{22} \mathrm{H}_{22} \mathrm{~N}_{2} \mathrm{O}_{3} ; \mathrm{C}, 72.91 ; \mathrm{H}, 6.12 ; \mathrm{N}, 7.73$. Found $\mathrm{C}$, 73.12; H, 5.94; N, 7.77 .

Ethyl 2-[[3-(4-chlorophenyl)-1-phenyl-1H-pyrazol-4-yl](hydroxy)methyl]acrylate (23c). White solid, mp 99-102 ${ }^{\circ} \mathrm{C} ; v_{\max }(\mathrm{KBr}) 1715\left(\mathrm{CO}_{2} \mathrm{Et}\right), 3399(\mathrm{OH}) \mathrm{cm}^{-1} ;{ }^{1} \mathrm{H} \mathrm{NMR}\left(\mathrm{CDCl}_{3}, 200\right.$ $\mathrm{MHz}) \delta=1.27\left(\mathrm{t}, 3 \mathrm{H}, J=7.2 \mathrm{~Hz}, \mathrm{CH}_{3} \mathrm{CH}_{2}\right.$ ), 3.06 (brs, $\left.1 \mathrm{H}, \mathrm{CHOH}\right), 4.22$ (q, 2H, $J=7.2 \mathrm{~Hz}$, $\left.\mathrm{CH}_{2} \mathrm{CH}_{3}\right), 5.76(\mathrm{~s}, 1 \mathrm{H}, \mathrm{CHOH}), 5.88\left(\mathrm{~s}, 1 \mathrm{H},=\mathrm{CH}_{2}\right), 6.36\left(\mathrm{~s}, 1 \mathrm{H},=\mathrm{CH}_{2}\right), 7.30-7.33(\mathrm{~m}, 1 \mathrm{H}, \mathrm{ArH})$, 7.39-7.50 (m, 4H, ArH), 7.71-7.77 (m, 4H, ArH), $7.95(\mathrm{~s}, 1 \mathrm{H},=\mathrm{CH})$; mass (ES+) $m / z=383.2$ $\left(\mathrm{M}^{+}+1\right)$, $385.2\left(\mathrm{M}^{+}+3\right)$. Anal Calcd. for $\mathrm{C}_{21} \mathrm{H}_{19} \mathrm{ClN}_{2} \mathrm{O}_{3} ; \mathrm{C}, 65.88 ; \mathrm{H}, 5.00 ; \mathrm{N}, 7.32$. Found $\mathrm{C}$, $66.06 ; \mathrm{H}, 5.23 ; \mathrm{N}, 7.15$.

tert-Butyl 2-\{hydroxy[3-(4-methylphenyl)-1-phenyl-1H-pyrazol-4-yl]methyl\}acrylate (24b). Yellow oil; $v_{\max }$ (Neat) $1709\left(\mathrm{CO}_{2} \mathrm{Et}\right), 3407(\mathrm{OH}) \mathrm{cm}^{-1} ;{ }^{1} \mathrm{H} \mathrm{NMR}\left(\mathrm{CDCl}_{3}, 200 \mathrm{MHz}\right) \delta=1.43(\mathrm{~s}$, $\left.9 \mathrm{H}, \mathrm{CCH}_{3}\right), 2.40\left(\mathrm{~s}, 3 \mathrm{H}, \mathrm{CH}_{3}\right), 3.10$ (brs, $\left.1 \mathrm{H}, \mathrm{CHOH}\right), 5.72(\mathrm{~s}, 1 \mathrm{H}, \mathrm{CHOH}), 5.80\left(\mathrm{~s}, 1 \mathrm{H},=\mathrm{CH}_{2}\right)$, $6.26\left(\mathrm{~s}, 1 \mathrm{H},=\mathrm{CH}_{2}\right), 7.23-7.75(\mathrm{~m}, 9 \mathrm{H}, \mathrm{ArH}), 7.94(\mathrm{~s}, 1 \mathrm{H},=\mathrm{CH})$; mass $(\mathrm{ES}+) \mathrm{m} / z=391.1\left(\mathrm{M}^{+}+1\right)$. Anal Calcd. for $\mathrm{C}_{24} \mathrm{H}_{26} \mathrm{~N}_{2} \mathrm{O}_{3} ; \mathrm{C}, 73.82 ; \mathrm{H}, 6.71 ; \mathrm{N}, 7.17$. Found $\mathrm{C}, 73.88 ; \mathrm{H}, 6.64 ; \mathrm{N}, 6.98$.

2-[Hydroxy(4-iodo-1,5-diphenyl-1H-pyrazol-3-yl)methyl]acrylonitrile (26a)- White solid, $\mathrm{mp}$ $148-150{ }^{\circ} \mathrm{C} ; v_{\max }(\mathrm{KBr}) 2229(\mathrm{CN}), 3398(\mathrm{OH}) \mathrm{cm}^{-1} ;{ }^{1} \mathrm{H} \mathrm{NMR}\left(\mathrm{CDCl}_{3}, 300 \mathrm{MHz}\right) \delta=3.43$ (brs, $1 \mathrm{H}, \mathrm{CHOH}), 5.49(\mathrm{~s}, 1 \mathrm{H}, \mathrm{CHOH}), 6.18\left(\mathrm{~d}, 1 \mathrm{H}, J=1.1 \mathrm{~Hz},=\mathrm{CH}_{2}\right), 6.30\left(\mathrm{~d}, 1 \mathrm{H}, J=1.1 \mathrm{~Hz},=\mathrm{CH}_{2}\right)$, 7.20-7.33 (m, 7H, ArH), 7.34-7.39 (m, 3H, ArH); ${ }^{13} \mathrm{C} \mathrm{NMR}\left(\mathrm{CDCl}_{3}, 75 \mathrm{MHz}\right) \delta=63.0,69.6$, $116.9,124.4,124.9,128.1,128.7,129.0,129.3,129.5,130.4,131.7,139.6,145.6,151.3$; mass (ES+) $m / z=428.0\left(\mathrm{M}^{+}+1\right)$. Anal Calcd. for $\mathrm{C}_{19} \mathrm{H}_{14} \mathrm{~N}_{3} \mathrm{O} ; \mathrm{C}, 53.41 ; \mathrm{H}, 3.30 ; \mathrm{N}, 9.84$. Found $\mathrm{C}$, $53.25 ; \mathrm{H}, 3.15 ; \mathrm{N}, 10.07$.

\section{2-\{Hydroxy[4-iodo-5-(4-methylphenyl)-1-phenyl-1 H-pyrazol-3-yl]methyl\}acrylonitrile} (26b). White solid, mp 150-152 ${ }^{\circ} \mathrm{C}$; $v_{\max }(\mathrm{KBr}) 2229(\mathrm{CN}), 3405(\mathrm{OH}) \mathrm{cm}^{-1} ;{ }^{1} \mathrm{H} \mathrm{NMR}\left(\mathrm{CDCl}_{3}\right.$, $300 \mathrm{MHz}) \delta=2.40\left(\mathrm{~s}, 3 \mathrm{H}, \mathrm{CH}_{3}\right), 3.35(\mathrm{~d}, 1 \mathrm{H}, J=7.2 \mathrm{~Hz}, \mathrm{CHOH}), 5.48(\mathrm{~d}, 1 \mathrm{H}, J=7.2 \mathrm{~Hz}$, $\mathrm{CHOH}), 6.19\left(\mathrm{~d}, 1 \mathrm{H}, J=1.0 \mathrm{~Hz},=\mathrm{CH}_{2}\right), 6.30\left(\mathrm{~d}, 1 \mathrm{H}, J=1.0 \mathrm{~Hz},=\mathrm{CH}_{2}\right), 7.15-7.33(\mathrm{~m}, 9 \mathrm{H}, \mathrm{ArH})$; mass $(\mathrm{ES}+) m / z=442.0\left(\mathrm{M}^{+}+1\right)$. Anal Calcd. for $\mathrm{C}_{20} \mathrm{H}_{16} \mathrm{IN}_{3} \mathrm{O} ; \mathrm{C}, 54.44 ; \mathrm{H}, 3.65 ; \mathrm{N}, 9.52$. Found C, 54.40; H, 3.69; N, 9.73. 
2-[[5-(4-Chlorophenyl)-4-iodo-1-phenyl-1H-pyrazol-3-yl](hydroxy)methyl]acrylo-nitrile (26c). White solid, mp $148-151{ }^{\circ} \mathrm{C} ; v_{\max }(\mathrm{KBr}) 2231(\mathrm{CN}), 3406(\mathrm{OH}) \mathrm{cm}^{-1} ;{ }^{1} \mathrm{H} \mathrm{NMR}\left(\mathrm{CDCl}_{3}\right.$, $300 \mathrm{MHz}) \delta=3.31(\mathrm{brs}, 1 \mathrm{H}, \mathrm{CHOH}), 5.48(\mathrm{~s}, 1 \mathrm{H}, \mathrm{CHOH}), 6.19\left(\mathrm{~s}, 1 \mathrm{H},=\mathrm{CH}_{2}\right), 6.31(\mathrm{~d}, 1 \mathrm{H}, J=$ $\left.0.6 \mathrm{~Hz},=\mathrm{CH}_{2}\right), 7.20-7.39(\mathrm{~m}, 9 \mathrm{H}, \mathrm{ArH})$; mass $(\mathrm{ES}+) \mathrm{m} / z=462.0\left(\mathrm{M}^{+}+1\right)$. Anal Calcd. for $\mathrm{C}_{19} \mathrm{H}_{13} \mathrm{ClIN}_{3} \mathrm{O} ; \mathrm{C}, 49.43 ; \mathrm{H}, 2.84 ; \mathrm{N}, 9.10$. Found C, 49.22; H, 3.03; N, 8.87.

Preparation of 4-Iodo-1,5-diphenyl-1H-pyrazole-3-carbaldehyde. To a stirred solution of appropriate compound from $\mathbf{7 a - c}(4.03 \mathrm{mmol})$ in $\mathrm{CHCl}_{3}(30 \mathrm{~mL})$ was added $\mathrm{K}_{2} \mathrm{CO}_{3}(1.67 \mathrm{~g}, 12.1$ $\mathrm{mmol}$ ) followed by dropwise addition of a solution of iodine monochloride (1.97 $\mathrm{g}, 12.1 \mathrm{mmol})$ in $\mathrm{CHCl}_{3}(5 \mathrm{~mL})$. After $18 \mathrm{~h}$ of stirring at room temperature, the reaction was quenched with $\mathrm{Na}_{2} \mathrm{SO}_{3}(1.0 \mathrm{M}, 40 \mathrm{~mL})$ and extracted with $\mathrm{CH}_{2} \mathrm{Cl}_{2}(3 \times 30 \mathrm{~mL})$. The organic phases were pooled, dried over $\mathrm{Na}_{2} \mathrm{SO}_{4}$ and evaporated in vacuo. Purification of the crude product by column chromatography (silica gel 60-120 mesh) using EtOAc/hexane as the eluent gave the substituted 1,5-diphenyl-1 $H$-pyrazole-3-carbaldehydes.

4-Iodo-1,5-diphenyl-1 $\boldsymbol{H}$-pyrazole-3-carbaldehyde (25a). White solid, mp 154-156 ${ }^{\circ} \mathrm{C} ; v_{\max }$ (KBr) $1696(\mathrm{CHO}) \mathrm{cm}^{-1} ;{ }^{1} \mathrm{H}$ NMR $\left(\mathrm{CDCl}_{3}, 200 \mathrm{MHz}\right) \delta=7.19-7.42(\mathrm{~m}, 10 \mathrm{H}, \mathrm{ArH}), 10.10(\mathrm{~s}$, $1 \mathrm{H}, \mathrm{CHO})$; mass $(\mathrm{FAB}+) m / z=375\left(\mathrm{M}^{+}+1\right)$.

4-Iodo-5-(4-methylphenyl)-1-phenyl-1 $H$-pyrazole-3-carbaldehyde (25b). White solid, mp $142-144{ }^{\circ} \mathrm{C} ; v_{\max }(\mathrm{KBr}) 1695(\mathrm{CHO}) \mathrm{cm}^{-1} ;{ }^{1} \mathrm{H}$ NMR $\left(\mathrm{CDCl}_{3}, 300 \mathrm{MHz}\right) \delta=2.40\left(\mathrm{~s}, 3 \mathrm{H}, \mathrm{CH}_{3}\right)$, 7.15-7.36 (m, 9H, ArH), ), $10.10(\mathrm{~s}, 1 \mathrm{H}, \mathrm{CHO}) ;{ }^{13} \mathrm{C} \mathrm{NMR}\left(\mathrm{CDCl}_{3}, 75 \mathrm{MHz}\right) \delta=21.5,62.2,125.0$, $125.5,128.7,129.1,129.5,129.6,129.8,130.3,139.5,139.8,147.0,149.0186 .1$; mass (ES+) $m / z=389.2\left(\mathrm{M}^{+}+1\right)$.

5-(4-Chlorophenyl)-4-iodo-1-phenyl-1H-pyrazole-3-carbaldehyde (25c). White solid, $\mathrm{mp}$ $156-158{ }^{\circ} \mathrm{C} ; v_{\max }(\mathrm{KBr}) 1694(\mathrm{CHO}) \mathrm{cm}^{-1 ; 1} \mathrm{H} \mathrm{NMR}\left(\mathrm{CDCl}_{3}, 200 \mathrm{MHz}\right) \delta=7.18-7.40(\mathrm{~m}, 9 \mathrm{H}$, $\mathrm{ArH}), 10.08$ (s, 1H, CHO); mass (ES+) $m / z=409.2\left(\mathrm{M}^{+}+1\right)$.

\section{Acknowledgements}

Two of the authors (SN and VS) gratefully acknowledge the financial support from UGC and CSIR, N. Delhi in the form of fellowship. This work, CDRI Comm. No.-7177, was supported by a DST-grant.

\section{References and Notes}

1. (a) Basavaiah, D.; Rao, A. J.; Satyanarayana, T. Chem. Rev. 2003, 103, 811. (b) Pathak, R.; Madapa, S.; Batra, S. Tetrahedron 2007, 63, 451. (c) Nag, S.; Yadav, G. P.; Maulik, P. R.; Batra, S. Synthesis 2007, 911. (d) Radha Krishna, P.; Narsingam, M. J. Comb. Chem. 2007, 9, 62. (e) Zhang, Q.; Sun, S.; Hu, J.; Liu, Q.; Tan, J. J. Org. Chem. 2007, 72, 139. (f) Declerck, V.; Allouchi, H.; Martinez, J.; Lamaty, F. J. Org. Chem. 2007, 72, 1518. (g) Chen, P. -Y.; Chen, H. -M.; Chen, L. -Y.; Tzeng, J. -Y.; Tsai, J. -C.; Chi, P. -C.; Li, S. -R.; Wang, 
E. -C. Tetrahedron 2007, 63, 2824. (h) Lee, K. Y.; Lee, H. S.; Kim, J. N. Tetrahedron Lett. 2007, 48, 2007. (i) Park, D. Y.; Lee, K. Y.; Kim, J. N. Tetrahedron Lett. 2007, 48, 1633. (j) Kim, S. J.; Lee, H. S.; Kim, J. N. Tetrahedron Lett. 2007, 48, 1069. (k) Majhi, T. P.; Neogi, A.; Ghosh, S.; Mukherjee, A. K.; Chattopadhyay, P. Tetrahedron 2006, 62, 12003. (1) Lee, K. Y.; Gowrisankar, S.; Lee, Y. J.; Kim, J. N. Tetrahedron 2006, 62, 8798. (m) Vasudevan, A.; Tseng, P. -S.; Djuric, S. W. Tetrahedron Lett. 2006, 47, 8591. (n) Shanmugam, P.; Vaithiyanathan, V.; Viswambharan, B. Tetrahedron 2006, 62, 4342. (o) Basavaiah, D.; Reddy, R. J.; Rao, J. S. Tetrahedron Lett. 2006, 47, 73. (p) Kim, S. C.; Lee, H. S.; Lee, Y. J.; Kim, J. N. Tetrahedron Lett. 2006, 47, 5681. (q) Park, H.; Cho, C. -W.; Krische, M. J. J. Org. Chem. 2006, 71, 7892. (r) Singh, V.; Saxena, R.; Batra, S. J. Org. Chem. 2005, 70, 353. (s) Ramesh, E.; Kathiresan, M.; Raghunathan, R. Tetrahedron Lett. 2007, 48, 1835. (t) Li, J.; Wang, X.; Zhang, Y. Tetrahedron Lett. 2005, 46, 5233. (u) Krafft, M. E.; Haxell, T. F. N. J. Am. Chem. Soc. 2005, 127, 10168.

2. (a) Gausepohl, R.; Buskens, P.; Kleinen, J.; Bruckmann, A.; Lehmann, C. W.; Klankermayer, J.; Leitner, W. Angew. Chem. Int. Ed. 2006, 45, 3689. (b) Davies, H. J.; Ruda, A. M.; Tomkinson, N. C. O. Tetrahedron Lett. 2007, 48, 1461.

3. (a) You, J.; Xu, J.; Verkade, J. G. Angew. Chem. Int. Ed. 2003, 42, 5054. (b) Tang, H.; Gao, P.; Zhao, G.; Zhou, Z.; He, L.; Tang, C. Catal. Comm. 2007, doi: 10.1016/j.catcom.2007.02.015. (c) Nakano, A.; Takahashi, K.; Ishihara, J.; Hatakeyama, S. Org. Lett. 2006, 8, 5357. (d) Berkessel, A.; Roland, K.; Neudorfl, J. M. Org. Lett. 2006, 8, 4195. (e) Pereira, S. I.; Adrio, J.; Silva, A. M. S.; Carretero, J. C. J. Org. Chem. 2005, 70, 10175. (f) Zhao, G. -L.; Shi, M. J. Org. Chem. 2005, 70, 9975. (g) Wang, J.; Li, H.; Yu, X.; Zu, L.; Wang, W. Org. Lett. 2005, 7, 4293. (h) Matsui, K.; Takizawa, S.; Sasai, H. J. Am. Chem. Soc. 2005, 127, 3680. (i) Reetz, M. T.; Mondière, R.; Carballeira, J. D. Tetrahedron Lett. 2007, 48, 1679. (j) Vasbinder, M. M.; Imbriglio, J. E.; Miller, S. J. Tetrahedron 2006, 62, 11450. (k) Mi, X.; Luo, S.; Cheng, J. -P. J. Org. Chem. 2005, 70, 2338.

4. (a) Aggarwal, V. K.; Fulford, S. Y.; Lloyd-Jones, G. C. Angew. Chem. Int. Ed. 2005, 44, 1706. (b) Santos, L. S.; Pavam, C. H.; Almeida, W. P.; Coelho, F.; Eberlin, M. N. Angew. Chem. Int. Ed. 2004, 43, 4330. (c) Teng, W. -D.; Huang, R.; Kwong, C. K. -W.; Shi, M.; Toy, P. H. J. Org. Chem. 2006, 71, 368. (d) Price, K. E.; Broadwater, S. J.; Walker, B. J.; McQuade, D. T. J. Org. Chem. 2005, 70, 3980. (e) Price, K. E.; Broadwater, S. J.; Jung, H. M.; McQuade, D. T. Org. Lett. 2005, 7, 147. (f) Buskens, P.; Klankermayer, J.; Leitner, W. J. Am. Chem. Soc. 2005, 127, 16762. (g) Shi, M.; Chen, L. -H.; Li, C. -Q. J. Am. Chem. Soc. 2005, 127, 3790 .

5. (a) Patra, A.; Batra, S.; Kundu, B.; Joshi, B. S.; Roy, R.; Bhaduri, A. P. Synthesis 2001, 276.

(b) Roy, A. K.; Batra, S. Synthesis 2003, 1347. (c) Roy, A. K.; Batra, S. Synthesis, 2003, 2325 .

6. Batra, S.; Roy, A. K. Synthesis 2004, 2550.

7. Narender, P.; Srinivas, U.; Gangadasu, B.; Biswas, S.; Rao V. J. Bioorg Med. Chem. Lett. 2005, 15, 5378. 
8. (a) Elguero, J. Comprehensive Heterocyclic Chemistry; ed. by Katritzky, A. R., Rees, C. W., Scriven, E. F. V.; Pergamon: Oxford, 1996; Vol. 5. (b) Elguero, J.; Goya, P.; Jagerovic, N.; Silva, A. M. S. Targets Heterocycl. Syst. 2002, 6, 52. (c) Penning, T. D.; Talley, J. J.; Bertenshaw, S. R.; Carter, J. S.; Collins, P. W.; Docter, S.; Graneto, M. J.; Lee, L. F.; Malecha, J. W.; Miyashiro, J. M.; Rogers, R. S.; Rogier, D. J.; Yu, S. S.; Anderson, G. D.; Burton, E. G.; Cogburn, J. N.; Gregory, S. A.; Koboldt, C. M.; Perkins, W. E.; Seibert, K.; Veenhuizen, A. W.; Zhang, Y. Y.; Isakson, P. C. J. Med. Chem. 1997, 40, 1347. (d) Terrett, N. K.; Bell, A. S.; Brown, D.; Ellis, P. Bioorg. Med. Chem. Lett. 1996, 6, 1819. (e) Takagi, K.; Tanaka, M.; Murakami, Y.; Morita, H.; Aotsuka, T. Eur. J. Med. Chem-Chim. Ther. 1986, 21, 65. (f) Genin, M. J.; Biles, C.; Keiser, B. J.; Poppe, S. M.; Swaney, S. M.; Tarpley, W. G.; Yagi, Y.; Romero, D. L. J. Med. Chem. 2000, 43, 1034. (g) Stauffer, S. R.; Coletta, C. J.; Tedesco, R.; Nishiguchi, G.; Carlson, K.; Sun, J.; Katzenellenbogen, B. S.; Katzenellenbogen, J. A. J. Med. Chem. 2000, 43, 4934. (h) Huang, Y. R.; Katzenellenbogen, J. A. Org. Lett. 2000, 2, 2833. (i) Kees, K. L.; Fitzgerald, J. J.; Steiner, K. E.; Mattes, J. F.; Mihan, B.; Tosi, T.; Mondoro, D.; McCaleb, M. L. J. Med. Chem. 1996, 39, 3920. (j) Sakya, S. M.; DeMello, K. M. L., Minich, M. L.; Rast, B.; Shavnya, A.; Rafka, R. J.; Koss, D. A.; Cheng, H.; Li, J.; Jaynes, B. H.; Ziegler, C. B.; Mann, D. W.; Petras, C. F.; Seibel, S. B.; Silvia, A. M.; George, D. M.; Lund, L. A.; Denis, S. St.; Hickman, A.; Haven, M. L.; Lynch, M. P. Bioorg. Med. Chem. Lett. 2006, 16, 288. (k) Cheng, H.; DeMello, K. M. L.; Li, J.; Sakya, S. M.; Ando, K.; Kawamura, K.; Kato, T.; Rafka, R. J.; Jaynes, B. H.; Ziegler, C. B.; Stevens, R.; Lund, L. A.; Mann, D. W.; Kilroy, C.; Haven, M. L.; Nimz, E. L.; Dutra, J. K.; Li, C.; Minich, M. L.; Kolosko, N. L.; Petras, C.; Silvia, A. M.; Seibel, S. B. Bioorg. Med. Chem. Lett. 2006, 16, 2076. (1) Tanitame, A.; Oyamada, Y.; Ofuji, K.; Fujimoto, M.; Suzuki, K.; Ueda, T.; Terauchi, H.; Kawasaki, M.; Nagai, K.; Wachi, M.; Yamagishi, J. -I. Bioorg. Med. Chem. 2004, 12, 5515. (m) Jia, Z. J.; Wu, Y.; Huang, W.; Zhang, P.; Song, Y.; Woolfrey, J.; Sinha, U.; Arfsten, A. E.; Edwards, S. T.; Hutchaleelaha, A.; Hollennbach, S. J.; Lambing, J. L.; Scarborough, R. M.; Zhu, B. -Y. Bioorg. Med. Chem. Lett. 2004, 14, 1229. (n) Thomas, B. F.; Francisco, M. E. Y.; Seltzman, H. H.; Thomas, J. B.; Fix, S. E.; Schulz, A. -K.; Gilliam, A. F.; Pertwee, R. G.; Stevenson, L. A. Bioorg. Med. Chem. 2005, 13, 5463.

9. Singh, S. K.; Saibaba, V.; Rao, K. S.; Reddy, P. G.; Daga, P. R.; Rajjak, S. A.; Misra, P.; Rao Y. K. Eur. J. Med. Chem. 2005, 40, 977.

10. Coelho, F.; Almeida, W. P.; Veronese, D.; Mateus, C. R.; Silva Lopes, E. C.; Rossi, R. C.; Silveira, G. P. C.; Pavam, C. H. Tetrahedron 2002, 58, 7437.

11. Basavaiah, D.; Gowriswari, V. V. L.; Bharathi, T. K. Tetrahedron Lett. 1987. 28, 4591.

12. Rainer, G.; Karuger, U.; Klemn, K. Arzneim.-Forsch. I981, 31, 649.

13. Felding, J.; Kristensen, J.; Bjerregaard, T.; Sander, L.; Vedso, P.; Begtrup, M. J. Org. Chem.1999, 64, 4196.

14. Schnürch, M.; Flasik, R.; Khan, A. F.; Spina, M.; Mihovilovic, M. D.; Stanetty, P. Eur. J. Org. Chem. 2006, 3283 and references cited therein. 